\title{
Taxonomic revision of the genus Logfia (Asteraceae, Gnaphalieae) in the Mediterranean region
}

\author{
Santiago Andrés-Sánchez", M. Montserrat Martínez-Ortega \& Enrique Rico \\ Departamento de Botánica, Facultad de Biología, Universidad de Salamanca, E-37007 Salamanca, Spain \\ santiandres@usal.es; mmo@usal.es; erico@usal.es
}

\begin{abstract}
Andrés-Sánchez, S., Martínez Ortega, M.M. \& Rico, E. 2013. Taxonomic revision of the genus Logfia (Asteraceae, Gnaphalieae) in the Mediterranean region. Anales Jard. Bot. Madrid 70(1): 7-18.

The genus Logfia Cass. was described by Cassini in 1819 and has always been considered closely related to Filago L. The species of Logfia have been frequently included within Filago but, recently, based on morphological characters and DNA sequence analysis it has been demostrated that Logfia is more closely related to the American representatives of the tribe Filagininae than to the genus Filago and the idea that Filago and Logfia should be considered independent genera has been resurrected. In this work, the first complete taxonomic account for the western Mediterranean taxa belonging to Logfia is presented, based on the revision of more than 3400 sheets lodged at 32 herbaria. A detailed description of the genus and a key for the four species present in the study area are proposed. Descriptions of all species together with a complete nomenclatural treatment, as well as indications about habitat preferences, phenology, and geographic distribution are also provided. Additionally nine nomenclatural types are designated, among them, the lectotypes for the accepted names Logfia clementei (Willk.) Holub and Logfia minima (Sm.) Dumort. and the neotype of Logfia heterantha (Raf.) Holub.
\end{abstract}

Keywords: Filago group, chorology, Logfia clementei, L. gallica, L. heterantha, L. minima, nomenclature, taxonomy, typification.

\section{INTRODUCTION}

The genus Logfia Cass. was described by Cassini in 1819, based on Filago gallica L. The author carried out a detailed review of the genus Filago L. described by Linnaeus in 1753. In his study, Cassini split the genus Filago into six different taxonomic units [Filago, Gifola Cass., Ifloga Cass., Leontopodium Cass., Logfia and Oglifa (Cass.) Cass.], which were considered to be morphologically very similar. Thus, believing that they should be taxonomically very close he used mainly anagrams of the word Filago to name them. Traditionally these genera constitute the subtribe Filagininae (Bentham, 1873 ) or the Filago complex (Anderberg, 1991), a very intricated taxonomic group, characterized by a general scarcity of morphological traits traditionally considered useful to delimitate genera and species.

Furthermore, ancient hybridization has been proposed as the most plausible explanation for the incongruence found between phylogenies obtained using different inherited types of DNA (Galbany-Casals \& al., 2010), and could also be the cause for the apparent homoplasy or erratical distribution of some character states among the species included in the group. This has probably contributed additionally to obscure the limits among the genera that integrate the Filago complex.

In this regard, one of the most problematic cases is the limit drawn between Filago and Logfia. They have been considered

\section{Resumen}

Andrés-Sánchez, S., Martínez Ortega, M.M. \& Rico, E. 2013. Revisión taxonómica del género Logfia (Asteraceae, Gnaphalieae) en la región mediterránea. Anales Jard. Bot. Madrid 70(1): 7-18 (en inglés).

El género Logfia Cass. fue descrito por Cassini en 1819 y siempre se ha considerado muy relacionado con el género Filago L., incluso en ocasiones ha sido incluido en este género. Recientemente se ha demostrado, sobre la base de caracteres morfológicos y secuencias de ADN, que Logfia es un género independiente, más relacionado con los representantes americanos de la subtribu Filagininae que con el género Filago. Se ha llevado a cabo el primer estudio taxonómico completo del género Logfia en la región mediterránea, basado en la revisión de más de 3400 pliegos conservados en 32 herbarios. En este trabajo se presenta una descripción detallada del género y una clave de identificación de las cuatro especies presentes en el área de estudio. Se proporciona, además, una descripción para cada una de las especies junto con una completa revisión nomenclatural y un resumen acerca de su hábitat, fenología, distribución geográfica y los mapas correspondientes elaborados sobre la base del material estudiado. Asimismo se designan nueve tipos nomenclaturales, entre ellos los de los nombres aceptados Logfia clementei (Willk.) Holub y Logfia minima (Sm.) Dumort. y el neotipo Logfia heterantha (Raf.) Holub.

Palabras clave: complejo Filago, corología, Logfia clementei, L. gallica, L. heterantha, L. minima, nomenclatura, taxonomía, tipificación.

as two independent genera by some authors (Pomel, 1874; Holub, 1975, 1976; Devesa, 1987; Anderberg, 1991), while others have considered that Logfia should be included in Filago (Candolle, 1837; Willkomm, 1870; Smoljaninova, 1959; Wagenitz, 1969, 1980; Bolòs \& Vigo, 1996; Devesa, 2002; Greuter, 2008). Recently Galbany-Casals \& al. (2010) obtained the first phylogeny of the Filago group based in nuclear and plastid DNA sequence data. Two well supported lineages were recovered, one of them includes the genus Logfia together with the North American representatives of the Filago group and the other is composed by the Eurasian representatives of the complex excluding Logfia.

An additional problem is that related with the identity and delimitation of Oglifa. Since Cassini (1819) described the genera Logfia and Oglifa they have been considered either synonyms (Candolle, 1837; Battandier, 1888; Wagenitz, 1969, 1980; Anderberg, 1991), or two different taxa (Cosson \& Germain, 1843; Willkomm, 1870; Smoljaninova, 1959). The available data on DNA sequences (Galbany-Casals \& al., 2010) and genome size (Andrés-Sánchez \& al., 2013) support the idea that Oglifa and Logfia should be considered two independent taxonomic entities. In our opinion, Logfia should be treated as an independent genus, while Oglifa-composed of Filago arvensis L., F. griffithii (A. Gray) Andrés-Sánchez \& Galbany and F. paradoxa Wagenitz- meres the subgeneric

\footnotetext{
* Corresponding author.
} 
rank under Filago (Galbany-Casals \& al., 2010; AndrésSánchez \& al., 2011). According to several authors, (Smoljaninova, 1959; Wagenitz, 1969; Holub, 1976; Anderberg, 1991; Andrés-Sánchez \& al., 2011) Logfia comprises 4 to 7 species, widely distributed throughout Europe, Near East and North Africa. Some of them have been introduced in America, Oceania and other areas of Asia (Anderberg, 1991; GalbanyCasals \& al., 2010; Andrés-Sánchez \& al., 2011). Four species are represented in the Mediterranean region: Logfia clementei (Willk.) Holub, L. gallica (L.) Coss. \& Germ., L. beterantha (Raf.) Holub and L. minima (Sm.) Dumort.

The diagnostic characters that help to differentiate Logfia from the remainder genera of the Filago group are: first leaves of seedlings linear; involucre always present; external receptacular paleae enclosing female florets, which are coriaceous in fruit; corolla of the external female florets slightly laterally attached to a reniform achene, and achene always glabrous (Galbany-Casals \& al., 2010).

There is a single chromosome number known for the species of Logfia, i.e., $2 n=2 x=28$ (e.g., Fernandes \& Queirós, 1971; Watanabe, 2012; Andrés-Sánchez \& al., 2013).

The aim of this study is to elaborate a complete taxonomic account for the genus Logfia in the Mediterranean region, which includes detailed descriptions and complete nomenclatural treatments of all taxa, as well as an identification key to the species.

We also cite or provide types for the accepted name of each taxon, as well as for the corresponding most widely used synonyms.

\section{MATERIAL AND METHODS}

The overall taxonomic treatment accepted here is that proposed by Galbany-Casals \& al. (2010) and Andrés-Sánchez \& al. (2011).

We have studied more than 3400 sheets in order to get a precise idea of the morphological variability of the taxa in order to elaborate complete morphological descriptions, reliably delimit the geographical distribution of the species involved and provide typification of some names in order to fix their usage and precise application. The revised sheets are deposited in the following 32 herbaria or collections, listed by their acronyms according to Thiers (2012, continuously updated): ABH, ALME, B, BC, BCN, BM, C, COA, COI, COIWILLKOMM, G-DC, GDA-GDAC, GOET, JACA, JAEN, K, LINN, LPA, MA, MAF, MGC, MJG, MPU, MPUDELILE, MPU-MAIRE, P, P-LAMARCK, PO, SALA, SEV, W, WU. With the aim of completing the information obtained, we have visited several populations in their natural habitat. We also looked for new populations, mainly of those taxa with restricted or poorly known distribution areas. In total, we have visited more than 80 populations and, at least one sheet from each one was lodged in the herbarium SALA.

Due to the large amount of information obtained, it is shown here only partially. Thus, we provide a list of selected studied material (indication of the locality, collection date, collectors and herbarium number) after the description of each species. Whenever possible we have cited three localities corresponding to each country where the species is present.
For the Iberian Peninsula and the Balearic Islands, three sheets corresponding to each province were listed. This information is indicated in the general distribution of the taxon through abbreviations of the Spanish provinces and Portuguese regions according to the model used in Flora iberica (Castroviejo, coord. gen., 1986-2012). For some provinces there is no herbarium material supporting references published in the botanical literature and in these cases the provincial abbreviation is shown in brackets in the chorological summary. Finally we have prepared maps of the selected material with the software DIVA-GIS 7.5.0.

For the nomenclatural section we have examined more than 600 publications including articles, protologues and other taxonomic literature. We have found 45 names for the 4 species included in this study. For each species we have listed the accepted name and all the corresponding homotypic synonyms arranged chronologically, as well as the heterotypic synonyms ordered by date of publication of basionym. All names are accompanied by the author who proposed them together with the place and the date of publication. We have used the following abbreviations whenever necessary: "[basion.]" basyonim, "nom. inval." invalid name, o "nom. illeg." illegitimate name, followed by a reference to the relevant article of the International Code of Botanical Nomenclature (ICBN, McNeill \& al., 2006).

Following the group of names that share the same basionym we cite or provide their type. In each case, we mention the holotype, or the selected lectotype, or neotype. We have selected a lectotype according to Art. 9.2 from the latest edition of the ICBN (McNeill \& al., 2006) or a neotype (Art. 9.6 ICBN), whenever necessary. We only mention isotypes and syntypes when we have directly checked them.

\section{TAXONOMIC TREATMENT}

\section{Logfia Cass.}

Annual herbs, unarmed, densely hairy, with indumentum eglandular, villose-tomentose, grayish to gray-whitish, adpressed. Stems erect or ascending, unbranched, branched from the base or dichotomically branched in the inflorescence, in this case cymose in dichasium, pleochasium or monochasium. Leaves of the stem alternate, \pm patent to adpressed, linear to elliptic, entire, acute, with a small scarious brownish mucro, flat to undulate in the margin, sessile; involucrant leaves 2-7, in rosette surrounding the clusters, similar length or clearly longer than the capitula, linear to spathulate, entire, acute, with a small scarious brownish mucro, with the margin straight to undulate and flat to involute. Inflorescence in clusters or solitary capitula, arranged like to cymes: dichasium, pleochasium or monochasium, sometimes spiciform, lax to \pm contracted; clusters with 1-14 capitula, subglobose, lax, some terminals and other axillary. Capitula disciform, heterogamous, sessile or pedunculated, ovoid, with or without marked angles, villose-tomentose. Involucre of 3-5 phyllaries, narrowly lanceolate to elliptic, acute, scarious in fruit, green to brownish with hyaline margin, sometimes purple, glabrous to villose-tomentose on the abaxial face and glabrous on adaxial. Receptacle obconic. Receptacular paleae $8-12$, imbricate, apparently arranged in two whorls, \pm patent 
in fruit; external 3-7, ovate, navicular - deeply or completely enclosing a female floret placed in this axil-, acute to subacute, green with hyaline margin, sometimes purple at the tip, herbaceous in flower and coriaceous in fruit, glabrous to villose-tomentose in the abaxial face and glabrous on adaxial; internal 5-7, lanceolate to elliptic, slightly concave, surrounding together the inner florets, obtuse, green with hyaline margin, sometimes purple at the tip, herbaceous in flower and scarious in fruit, glabrous to villose-tomentose in the abaxial face and glabrous on adaxial. External florets 3-7, female, filiform, corolla slightly laterally attached, whitish-yellowish, with the tip reddish to brownish, entire or slightly lacerated; inner florets of two types; 3-20 female, filiform, similar to external florets but the corolla apically attached; and 2-8 hermafrodite florets, fully fertile, tubular, whitish-yellowish to greenish, with 4 tooths reddish. Anthers with basal appendages. Achenes heteromorphic; external reniform, usually compressed dorsiventrally, brown-olive green, glabrous; inner cylindrical to ellipsoid, slightly compressed dorsiventrally, brown-olive green, papillose. Pappus of the external florets absent and of the internal ones always present, with 12-25 white scabrid bristles.

\section{KEY TO THE SPECIES}

1. Involucrant leaves linear to linear-lanceolate, clearly longer than the capitula, with the margin involute; external receptacular paleae completely enclosing corresponding floret ........................ 1. L. gallica

1. Involucrant leaves lanceolate to elliptic or spathulate, similar length than the capitula, or sometimes longer than the capitula, with the margin flat, rarely slightly involute; external receptacular paleae completely or deeply enclosing corresponding florets .................................. 2

2. Capitula solitary, rarely 2-3 grouped together in clusters, pedunculated, rarely sessile; involucrant leaves with the margin slightly undulate; external receptacular paleae deeply enclosing corresponding floret .....

\section{L. heterantha}

2. Capitula in clusters of 1-7, sessile or rarely short pedunculated; involucrant leaves with the margin straight; external receptacular completely or deeply enclosing corresponding floret ........................................ 3

3. Capitula in clusters of $1-3$, with 5 highly marked angles; receptacular paleae 8-10, external completely enclosing corresponding florets; inner female florets 4-7

2. L. clementei

3. Capitula in clusters of 3-7, rarely solitary, with 5 slightly marked angles; receptacular paleae 10-12, external deeply enclosing corresponding floret; inner female florets 8-15

3. L. minima

\section{Species descriptions}

1. Logfia gallica (L.) Coss. \& Germ. in Ann. Sci. Nat., Bot. sér. II, 20: 291 (1843). Filago gallica L., Sp. Pl. 2: 1230 (1753), [basion.]. Gnaphalium gallicum L., Sp. Pl. 2: 857 (1753). Xerotium gallicum (L.) Bluff \& Fingerh., Comp. Fl. German. sect. I. 2:344 (1825). Oglifa gallica (L.) Chrtek \& Holub in Preslia 35: 10 (1963). Filago filiformis Lam., Fl. Franç. 2: 61 (1779) nom. illeg. (Art. 52.1). Ind. loc.: "Habitat in Anglia, Gallia". (Lectotype, designated by Alavi, 1983; LINN 1041.6!).

Filago tenuifolia C. Presl, Delic Prag. 1: 101 (1822). Filago gallica var. tenuifolia (C. Presl) DC., Prodr. 6: 248 (1838). Filago gallica subsp. tenuifolia (C. Presl) Arcang., Comp. Fl. Ital.: 379 (1882). Logfia tenuifolia (C. Presl.) H. J. Coste, Fl. Descr. France 2: 326 (1903). Ind. loc.: "Hab. in arenosis montium Messinam Siciliae cingentium". (Type not found in PR, PCR and BRNM).
Logfia subulata Cass. in F. Cuvier, Dict. Sci. Nat. ed. 2, 27: 117 (1823). Ind. loc.: “ ... champs sablonneux des environs de Paris ...". (Lectotype, here designated, P! Herbier M. de Jussieu).

Filago germanica var. longibracteata Willk. in Bot. Zeitung (Berlin) 5: 859 (1847). Filago gallica var. longibracteata (Willk.) Willk. in Willk. \& Lange, Prodr. Fl. Hispan. 2: 56 (1870). Ind. loc.: "Hab. in Castella nova circa oppidum Chamartin et in ditione la Moralesa". (Lectotype, here designated, COI 00035403!).

Filago tenuifolia var. multicaulis Foucaud \& E. Simon in Trois semaines d'herborisations en Corse. Note 15: 180 (1898). Filago gallica var. multicaulis (Foucaud \& E. Simon) Rouy in Rouy, Foucaud \& E. G. Camus, Fl. France 8: 179 (1903). Ind. loc.: "près d'Ajaccio". (Lectotype, here designated, MPU! Herbier E. Simon Corse).

Filago tenuifolia var. nana Rouy in Rouy, Foucaud \& E. G. Camus, Fl. France 8: 179 (1903). Ind. loc.: "iles de Lavezzi". (Type not found).

Filago tenuifolia var. simplex Foucaud \& E. Simon in Trois semaines d'herborisations en Corse. Note 15: 179 (1898). Filago gallica var. simplex (Foucaud \& E. Simon) Rouy in Rouy, Foucaud \& E. G. Camus, Fl. France 8: 179 (1903). Ind. loc.: "Calvi, Corse/ 14-may-1896/ Foucaud". (Lectotype, here designated, LY-F39S photo!).

Logfia gallica subsp. insularis Holub in sched. (1978), nom. inval. (Art. 32.1). Type: K 37397 !, BM 000907403 !.

Iconographies. Cosson \& Germain (1843: 293, Pl. 13 Fig. A); Reichenbach f. (1854: 36 lam. CMXLVII, fig. I); Valdés, Talavera \& Galiano (eds.) (1987: 26); Fig. 1a; Fig. 2 s-v.

Annual herb densely hairy, with indumentum grayish to gray-whitish. Stems $2-33 \mathrm{~cm}$, branched or unbranched. Leaves of the stem $8-28 \times 0.2-2 \mathrm{~mm}$, adpressed to \pm patent, linear to linear-lanceolate, flat in the margin; involucrant leaves 4-5, 4-26 × 0.2-2 mm, clearly longer than the capitula, linear to linear-lanceolate, with the margin straight and involute. Inflorescence in clusters arranged like to dichasium or pleochasium, some of them like to monochasium, usually lax; clusters with (1)3-14 capitula, lax. Capitula 3.5-5 × 2.5-3 mm, sessile or rarely short pedunculated, with 5 marked angles. Phyllaries 2-2.5 $\times 0.5-1 \mathrm{~mm}$, lanceolate to elliptic, green to brownish with hyaline margin, rarely purple at the tip, glabrous to villose-tomentose on the abaxial face. Receptacular paleae 10-12; external 5, 3.5-4 × 1-1.5 mm, completely enclosing the external female floret, acute to subacute, green with hyaline margin, sometimes purple at the tip, coriaceous in fruit, glabrous to villose-tomentose in the abaxial face; internal 5-7, 3-4 $\times 0.8-1,5 \mathrm{~mm}$, surrounding together the inner florets, obtuse, green with hyaline margin, sometimes purple at the tip, scarious in fruit, glabrous to villose-tomentose in the abaxial face. External florets 5, 2-3 mm, female; inner florets of two types, 8-17 female similar to external florets and 26 hermafrodite of $2.5-3 \mathrm{~mm}$. Achenes of the external florets 0.5-1.1 $\times 0.2-0.5 \mathrm{~mm}$; inner 0.4-0.7 $\times 0.2-0.5 \mathrm{~mm}$. Pappus of the internal florets with $20-25$ bristles. $2 n=28$.

Habitat, phenology and distribution (Fig. 3). Ruderal, road margins, fallow lands, culture edges, stony areas, forest or 

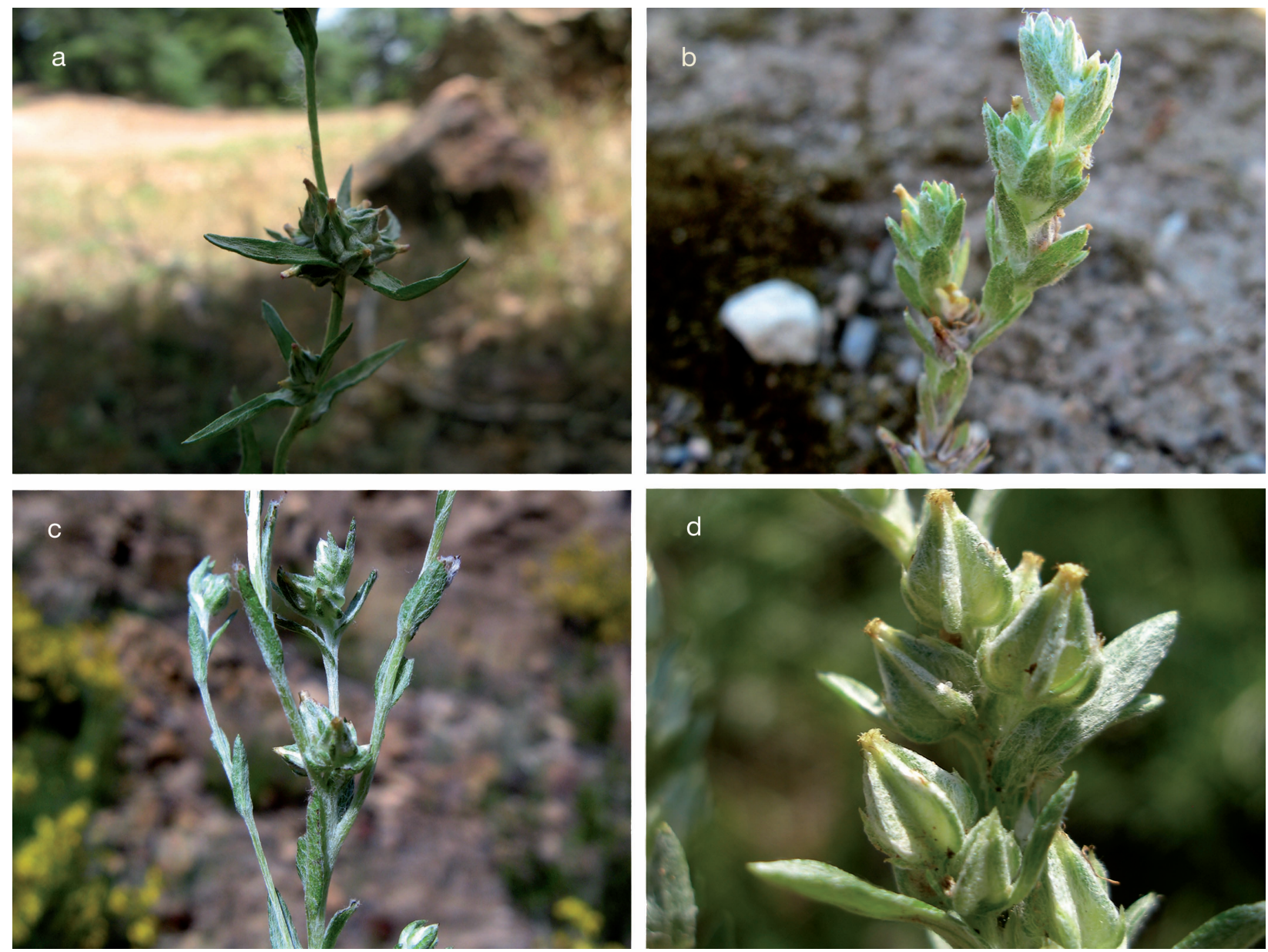

Fig. 1. a, Logfia gallica (Djebel Tazzeka, Morocco); b, L. clementei (Níjar, Almería, Spain); c, L. minima (Garcibuey, Salamanca, Spain); d, L. heterantha (Djurdjura, Algeria).

scrub gaps, salt marshes, dry riverbeds, soil indifferent; 0 1800 m. III-VIII. Europe, Macaronesian Islands, N of Africa and SW of Asia, introduced in the United States, Chile and India. Distributed almost throughout the Iberian Peninsula and the Balearic Islands. Esp.: A Ab Al Av B Ba Bi Bu C Ca Cc Co CR Cs Ge Gr HJ Le Lo Lu M Ma Mu Na O Or (P) PM Po S Sa Se Sg So SS T (Te) To V Va ViZZa. Port.: AAl Ag BA BAl BB BL DL E Mi R TM. N.v.: yerba para las calenturas; port.: erva do picanço.

Observations. Willkomm (1847) described Filago germanica var. longibracteata Willk. from the Iberian Peninsula and later (Willkomm, 1870) he transferred this variety to Filago gallica. The diagnostic characters proposed for this taxon were: weaker stems covered by a less dense indumentum than that shown by the nominated variety, and involucral leaves more than three times longer than clusters. However, neither morphological characters nor distribution areas allow us to clearly discriminate between the two taxa. A detailed study of these characters from both, herbarium and fresh material, together with the consideration of further diagnostic traits, have showed that the characters used by Willkomm to describe the var. longibracteata are within the range of variability of the species. This species is very polymorphic and strongly influenced by the environmental conditions and anthropic pressure.

\section{Selected material}

ALBANIA: Vallona, ad pagum Pogdania prope Suernec, 29-VI-1894, $A$. Baldacci (K, P). ALGERIA: Gran Kabilia, $M^{\circ}$ Djurjura, commune de Aim Hamma, 19-VI-2010, M.M. Martínez-Ortega, E al. MO5520 (SALA 139152); Alger, collines, V-1839, N. Bové (K); Alger, près d'Alger, est de la forêt de Réghaïa, 24-IV-1960, A. Dubuis (MPU DUBUIS). CHILE: prope la Quinta, in declivibus, asperisque collium Quillota, IX/X-1828/1829, Unio itinere Hrbr. Bertero nr.; Herb. Schultz Bip. (P). CROATIA: Istriae, ad oppidum Pola, VII-1877, J. Freyn - Dr. C. Baenitz, Herbarium Europaeum (GOET Old Herbarium, WU-Hal-Eur); Medolino Istria, VI, T. Pichler (MPU). CYPRUS: Larnaka, Stavrovouni, 15-IV-1991, 429 OPTIMA, Iter Medit. IV (vgl. Bocconea 11: 35. 1999) (GOET); Region 1, bei Meikle, Akamas-Halbinsel, am Smieyes-(Smigies) Naturlehrpfad westl. Neo Chorion, 24-IV-1999, G. Wagenitz 4996 (GOET); Bei Kalapanayiotis (NNW Troodos), 19-IV-1999, G. Wagenitz 4942 (GOET). FRANCE: Corse, Solenzara, Iemkies, 16-VII1932, P. Aellen - Flora von Corsica 475 (K); Hérault, Roquehaute, VI-1954, Herbier Albaille (MPU); Pyrénées-Orientales, östl. vom Canigou, zwischen Fourques u. Llauro, 8-VI-1974, G. Wagenitz 2569 (GOET). GERMANY: Saarbrück, Saalfelder, 36 Herbarium W. de Schoenefeld (MPU); Baden, Karlsruhe (GOET); Neuburg, Herb. Vocke (GOET Old Herbarium). GREECE: Thessalia, Caterinam (prope), 12/24-VII-1857, T. G. Orphanides (WU-Hal-Graec); Dodecanese, Patmos, in short turf on a headland, SW coast of the island due W of the Monastery, 6-IV-1969, C.C. Townsend-Flora of Greece 69/62 (K); Creta, Creta occidentalis au Ennea Horia, VI-1846, Herb. De Heldreich (K). INDIA: Sem Padum a Balbis, 1843, Herb. Schultz Bip. (P). ISRAEL: Herzlia, near Tel-Aviv, 26-IV-1929, A. Eig \& al. 94 (MPU, MAF 57529). ITALY: Sicilia, Ficuzza, F.J. Lagger-Herb. Vocke (GOET Old Herbarium); Cerdeña, Nuoro Perdasdefogu, carretera hacia Esterzili, 7-VI- 

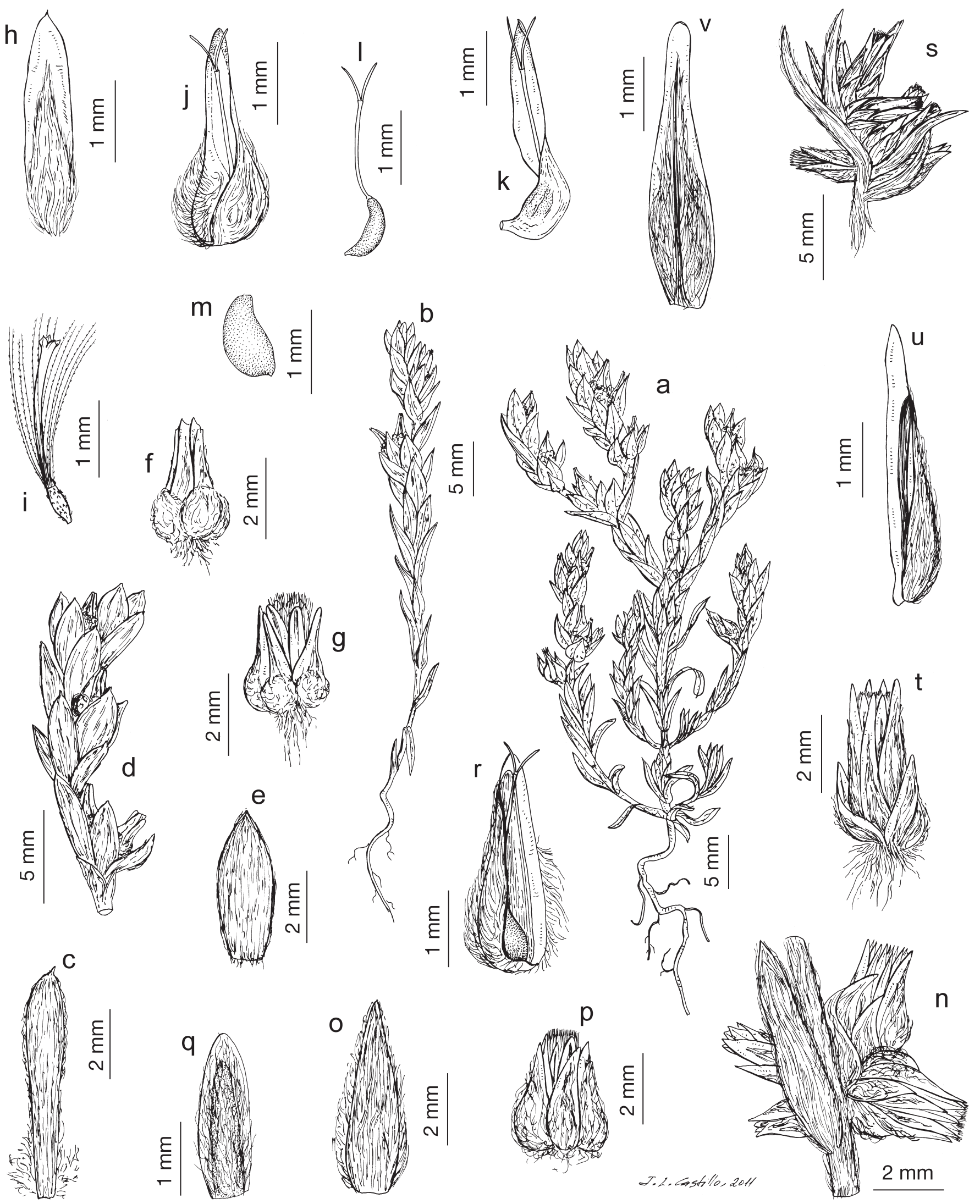

Fig. 2. Logfia clementei, Garrobillo, Águilas, Murcia (SALA 139202): a, b, habit; c, stem leaf; d, inflorescence; e, involucrant leaf; f, g, capitulum, without phyllaries; $\mathbf{h}$, phyllary; i, hermafrodite floret; $\mathbf{j}, \mathbf{k}$, receptacular paleae and female floret; $\mathbf{I}$, external female floret; $\mathbf{m}$, achene of external female floret. L. minima, Villarino de los Aires, Salamanca (SALA 134221): n, cluster; o, involucrant leaf; p, capitulum, without external phyllaries; $\mathbf{q}$, phyllary; $\mathbf{r}$, receptacular paleae and external female floret. L. gallica, entre Breda y Sant Celoni, Barcelona (SALA 139203): s, cluster; t, capitulum, with phyllaries; u, external receptacular paleae, lateral view; v, external receptacular paleae, abaxial view. 


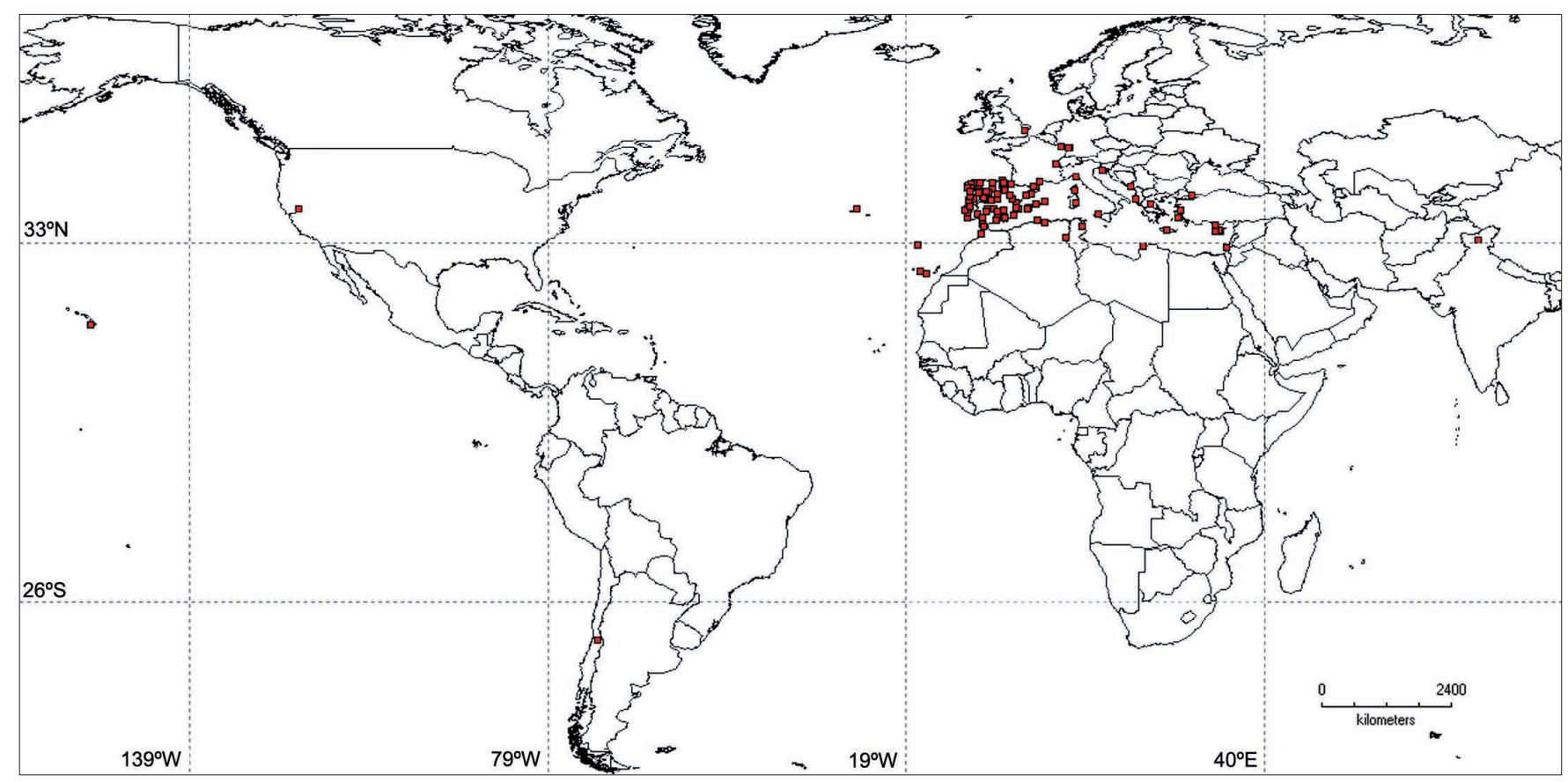

Fig. 3. Distribution map of the selected studied material corresponding to Logfia gallica.

2003, C. Aedo E al. MAG3805 (SALA 139155); Riviera di Levante, Commune di Tramura, tuto of hill above Anzo, 12-VII-1964, B. Verbourt 4104 (K) LIBYA: Cyrenaica, Barce, 14-IV-1939, N. Y. Sandwith - Expedition to Libya 2629 (K). MONTENEGRO: Cataro, 3-VI-67, Huter (K). MOROCCO: Tánger-Tetuán, Tanger, carretera al Cabo Espartel, 5-VII-2010, V. Lucía, E al. DP767 (SALA 110262); Costa Atlantica, Kenitra, entre Tánger y Kenitra, Moulay Bouselham, 16-VI-2008, R. Casimiro-Soriguer \& al. 30/08 (SEV 227722). Plateau central, El-Menzeh, $10 \mathrm{~km}$ à l'W d'El-Khatnake, 28-V1969, J. Mathez 4863 (MPU MATHEZ). PORTUGAL: Algarve: Monchique, VI-1887, A. Möller (COI 00020594); Olhao, marismas del Puerto, 13IV-1996, E. Camuñas \& al. (ABH 30873); Olhao, Quinta de Marin, 7-VI1988, A. Moura (MA 479014). Alto Alentejo: Vendas-Novas, Vale de Águias, 13-V-1947, A. Fernandes E Sousa 2225 (COI 00020593); Évora, V-1891, A. Möller (COI 00020622); Alto da Queijeira: Serra d'Ossa, 7-V-1947, A. Fernandes E Sousa 1769 (COI 00020641). Azores: ad rupis, vias insularis, VI1838 (K); communicated from the Island of St. Michael, T. C. Hunt-Esq., British Consul in the Azores (P); Azores, 1857, P. Lawen 1720 (GOET). Baixo Alentejo: Santiago do Cacem, na Seara, 9-VI-1960, A. Fernandes, R. Fernandes E J. Matos 7310 (COI 00020640); Cabo de Sines, 25-V-1978, J.A. Devesa \& al. (SEV 35840). Beira Alta: Algodres, Cortiçô d'Algodres, VIII-1892, M. Ferreira (COI 00020614); Corregal do Sal Gavimhes, estrada Iliveira do Hospital, 17-VII-1975, Dramonde E al. 13503 (COI 00020635); Caramulo, na estrada, 31-VII-1944, J. Castro (PO 4245). Beira Baixa: Vovas de Boi, Serra da Estrela, VIII-86, J. Henriques (COI 00020605). Beira Litoral: Buarcos, VI-1869, E. Schmitz (MA 537062); Coimbra, Barconço, VI-1895, M. Ferreira (COI 00020596); Aveiro, Ilhavo, hacia Gafalha de Nazaré, 19-V-2009, E. Rico E al. SA295 (SALA 139153). Douro Litoral: Serra da Freita, 8-VII1966, A.C. Matos \& A. Dinis 9704 (COI 00020654). Estremadura: Alfarim, V-1882, A. Möller (COI 00020609); Alenquer, Montegil, VI-1892, A. Möller (COI 00020617); Serra do Montejunto, entre el radar y las ruinas del convento dominicano, 6-VI-2012, M.A. García \& al. MAG 6111 (SALA 145525). Madeira: in Corn fields in Sâo Vicente at the foot of the montains of Boa Ventura, 30-VII-1827, 386 Herbarium of Rev. R. T. Lowe Received April 1875 (K); Madeira, Pico da Silva, 28-V-1866, G. Mandon-Pl. Maderenses (P); Madeira, San Antonio da Serra, VII-1862, C.B. Clarke 86 (K). Minho: Fafe, Arnil, 20-VII-1941, P. Barros Carneiro (PO 30498); Sao Bento da Porta Aberta, Serra do Gerês, VI-1948 (MAF 79452); Arcos de Valle de Vez, VI-1890, A. Möller (COI 00020602). Ribatejo: Montargil, VI-1883, J. Corterao (COI 00020628); estrada Abrantes-Sardoal a 3 km do Sardoal, 18-VI-1956, A. Fernandes E al. 5863 (COI 00020606); São José da Lamarosa, monte do Leão, 8 VI-2012, A. Quintanar \& al. AQ4632 (SALA 145523). Tras-os-Montes: Mo- gadouro, carretera hacia Miranda, Serra do Variz, 19-5-1997, A. Pujadas \& L. Tejada (COA 23636); Carrazeda de Ansiâes, Arnedo, 11-VI-1942, A. Rozeira (PO 3331); Chaves, VII-1892, A. Möller (COI 00020615). SPAIN: Álava: Bernedo, Urturi, 13-VIII-1983, G. Morante E J.A. Alejandre (MA 422081); Maestu, Korres, 11-VIII-1986, J.A. Alejandre (MA 365855). Albacete: Villapalacios, 25-VI-1984, J.M ${ }^{a}$. Herranz (MA 319561); Sierra de Relumbrar, 21 VI-1986, J.M ${ }^{a}$. Herranz (MA 352189). Alicante: Tormos, prox. del pueblo, $27-$ IV-1995, J. X. Soler \& M. Signes JXS1693 (MA 561834); Altea, Sierra de Bérnia, 27-V-1959, A. Rigual (MA 369412, ABH 21431). Almería: carretera entre Macael y Tabernas, 20-IV-2008, S. Andrés-Sánchez \& S. Barrios de León SA171 (SALA 134255); Cuevas de Almanzora, Sierra Almagrera, 14-IV-2005, M.B. Crespo \& al. (ABH 48586); Benizalón, Cortijo de los Pajares, base de la Sierra de los Filabres, 9-V-1989, C. Morales \& A.B. Robles (GDAC 32080). Asturias: Corias, 29-VII-1952, E. Guinea (MA 444890); Cangas del Tineo, 19-VIII1835, M.C. Durieu 301 (MPU); Grado (alrededores), 10-VI-1944, O. Rodrí guez Sánchez (MAF 90963). Ávila: Candeleda, pantano del Rosarito, monte San Juan, 2-VI-1993, Aragón E Castillo GA0392 (MA 644792); Piedralaves, La Pinosilla, 4-VII-1984, P. Montserrat \& J.L. Gz. Rebollar (JACA 69484); Arenas de San Pedro, Río Pelayo, 10-VII-1988, I Aizpuru E al. ML28988bis (MA 451379). Badajoz: Almorchón, 29-VI-1952 (MAF 81834); Campanario, IV-1971, F.J. Fernández Casas (MA 422002); Herrera del Duque, 21-VI-1969, S. Rivas Goday \& M. Ladero (GDA 8310). Barcelona: entre Gavà y Berga, 4VI-1981, J.A. Devesa \& al. (SEV 64257); Barcelona, cunetera de les Aigües, Perdigó (BCN 35039); cerca de Batlloria, entre Breda y San Celoni, 17-VI2009, S. Andrés-Sánchez E al. SA321 (SALA 139203). Burgos: Aguillo, Condado de Treviño, 9-VII-1987, J.A. Alejandre (MA 423204); Cardeñajimeno, VII-VIII-1914, P. Font Quer (BC 31277); Valle de Mena, Tres Concejos, 30V-1999, P. Bariego E A. Gastón (SALA 104637). Cáceres: Cáceres, base del Portanchito, 16-V-1983, M. Ladero E M.T. Santos (SALA 66943); Alconétar, Riberos del Tajo, 24-V-1948, S. Rivas Goday \& J. Borja Carbonell (MAF 78371); Baños de Montemayor, 22-V-1944, A. Caballero (MA 124299). Cádiz: Conil de la Frontera, El Colorado, 17-VI-2009, E. Rico ER7963 (SALA 135582); Alcalá de los Gazules, S. ${ }^{a}$ del Algibe, El Picacho, 25-IV-1995, L. Lledó E al. (ABH 12813); entre Ubrique y Puerto de Galiz, 14-VII-1978, J.A. Devesa E al. (SEV 51049). Cantabria: Cabezón de Liébana, Riega Valdesusaña, pr. Vieda, 28-V-1996, G. Moreno Moral MM0113/1996 (MA 696988); Llano, Embalse del Ebro, 9-VII-2007, I. Aizpuru (GDA 53631); Cillorigo de Liébana, sobre Trillayo, 25-VII-1996, G. Moreno Moral E al. SP0619/1996 (MA 696987). Castellón: Segorbe, Monte Malo, V-1898, C. Pau (MA 124239); Chovar, Bco. del Carbo, Carret de Eslida, 8-VI-1989, P. Montserrat \& L. Villar (JACA 124589); Sierra de Espadán, in monte "La Rapita", 29-VI-1902, C. 
Pau (MA 448423). Ciudad Real: Almodóvar del Campo, La Garganta, Navarrillo, 22-VI-1997, R. García Rio (MA 596893); Fuencaliente, Puerto de Valderrepisa, 10-V-1997, P. Montserrat (JACA 47897); Calzada de Calatrava, Hoz del río Fresneda, 8-V-1995, J. Barrios Pérez E R. García Río (SALA 87506). Córdoba: S. ${ }^{a}$ Morena, prox. Arroyo Fresnedoso, 3-V-92, M. Melendo E E. Cano (GDAC 39249); Cardeña a Vilanueva, Km 16, 20-VI-2008, C. Fernández (JAEN 680178); de Fuenteobejuna a Cazalla, Km 25, 10-V-1983, A. Pujadas (COA 40352). Gerona: Alt Empordá, primavera 1993, I. Soriano E al. (BCN 35808); Vidreras, VII-1909, Xiberta Herbarium Font Quer (BC31281) La Jonquera, 16-VII-1993, C. Aedo \& al. M-H811 (MA 528952). Granada: Granada, Silla del Moro, 8-IV-1907, J.L. Diéz Tortosa (GDA 5022); S. a del Obeilar, 16-IV-1984, P. Aroza \& O. Socorro (GDA 16945); carretera de Murcia Km 266, 3-VI-1978, F. Valle (GDAC 10062). Guipúzcoa: Escoriaza, Barrio Mazmela, 28-VI-1973, P. Montserrat \& al. (JACA 233873); Escoriaza, (MA 124306); San Sebastián (alrededores), VII- ¿1875? (BC 31276). Huelva Sierra de Aracena, entre Aroche y Las Contiendas, 6-VI-1979, J. Rivera \& B Cabezudo 5058/R (SEV 48992); Huelva, 9-V-1903, C. Pau (MA 124277); Bollullos del Condado, 11-VII-1943 (GDA 5020). Islas Baleares: Mallorca, Xorrigo, 7-VI-1987, J. Vicens (BCN 26324); Menorca, Ciutadella, La Falconera, parte oriental, 12-V-1952, P. Montserrat (JACA 18152); Ibiza, Santa Eulalia des Riu, Puig de s'Argentera, 4-V-1996, A. Barber \& J.C. Cristóbal (ABH 33255). Jaén: de La Carolina al Centenillo, Sierra Morena, 19-IV-2006, M.M. Martínez-Ortega \& S. Andrés-Sánchez SA066 (SALA 134280); Despeñaperros, 5-V-1977, P. Montserrat (JACA 34577); Andújar, Casas del Lentisquillo, $27-$ V-1993, P. Díaz (MA 651859). La Coruña: Santiso, Barazón, 5-VII-2001, R.I Louzán E J. Rodríguez-Oubiña (SALA 108595); Betanzos, 21-IX-1851/52, J. Lange 253 (COI WILLKOMM COI 00035401); Santiso, Barazón, 5-VII 2001, R.I. Louzán E J. Rodriguez-Oubiña (ABH 48275). La Rioja: El Rasillo de Cameros, 17-VI, I. Zubía (MA 124288). Las Palmas: Valverde, Hierro, 22-V1901, J. Bornmüller 2490 (P); Tirajana, Gran Canaria, 3-III-1969, G. Kunkel 12519 (GOET); Tejeda (cerca de), Gran Canaria, 18-IV-1935, Cuatrecasas \& al. (MAF 62003). León: Villafranca del Bierzo, 2-VII-1852, J. Lange (MPU); Prada, 14-VII-1979, C. García González 38-Dt (JACA 88085). Lugo: Villardiaz-Fonsagrada, 20-VII-1953, E. Carreira (MA 167602). Madrid: in Castilla nova circa Chamartin et in ditione La Moraleja, 1844, H.M. Willkommii (COI WILLKOMM COI 00035403); cerca del Manzanares, 1799, J. D. Rodríguez (MA 124282); Madrid, V-1893, J. Mas y Guindal (MAF 63158). Málaga: Málaga, V-1968, Académia Malagueña C. (GDA 5024); prope Cueva de Nerja, 25-III-1978, R.M. Burton (SEV 35329); entre Álora y Carratraca, en la sierra de Aguas, 26-V-1983, E. Bayón É al. (MA 444699). Murcia: Cartagena, Hacienda La Baña, 9-V-1909, F.A. Ibáñez (MAF 57531); La Unión, Murcia, Valle de Escombreras, cala del Gorguel, 29-III-1998, C. Navarro \& al. CN1961 (MA 613678); Cartagena, Hacienda La Baña, 9-V-1909, F.A. Ibáñez (MAF 57532). Navarra: Garralda, 21-VII-1987, J. E G. Montserrat (JACA 0799687) Orense: Castrelo de Miño, 17-VII-1935, A. Rodríguez (MA 124308); Castrelo de Miño, Sierra de Santa Marta, 18-VII-1935, A. Rodríguez (MA 180047); Verín, bajada a Verín desde la autopista, 18-V-2009, E. Rico \& al. SA288 (SALA139154). Palencia: [Losa \& Montserrat in Anales Inst. Bot. Cavanilles 10(2): 413-509 (1953)]. Pontevedra: Sotomayor, proximidades del pueblo, VIII-1977, C. Morales (GDAC 3731); Marín, 20-VIII-1932, Gz. Albo (MA 124312); Bueu, Menduiña, 25-VI-1971, S. Castroviejo (MA 197292). Salamanca: Doñinos de Ledesma, Finca Muñovela, 19-V-1973, P. Montserrat \& L Villar (JACA 103373); Garcibuey, El Estanque, 31-V-2009, S. Andrés-Sánchez SA305 (SALA 135580); Ciudad Rodrigo, 3-VI-1976, E. Rico (MA 242813). Santa Cruz de Tenerife: San Diego del Monte, Tenerife, VI-1846, E. Bourgeau 435 (P); la Gomera, Bco. S. Sebastiano, 23-V-1894, R.P. Murray (K); Tenerife, P. Orotava, Barr. De Martianez, 27-III-58, Herbarium of Rev. R. T. Lowe Received April 1875 (K). Segovia: Cerezo de Arriba, Valhondo, 8-VII-1983, T. Romero (MA 568482); Cerezo de Abajo, 18-VI-1983, T. Romero (SALA 39633); Cerezo de Abajo, La Dehesa, 5-VI-1983, T. Romero (SALA 39634). Sevilla: Castillo de los Guardas, V-1914, Cogolludo (MA 124259); entre Bollullos y Aznalcázar, 3-VI-1971, E. Domínguez \& S. Talavera 1124/71 (SEV 9447); entre Puebla del Río y Venta de la Cruz, 15-V-1971, E.F. Galiano \& B Valdés 873/71 (SEV 9378). Soria: Valonsadero, 14-VI-1963, A. Segura Zubizarreta (MA 352890); Pinar de Cabrejas, 17-VII-1958, A. Segura Zubizarreta (MA 352989); Montenegro de Cameros, 20-VI-1925, A. Caballero (MA 124290). Tarragona: Coll d'Alforja, 31-V-1975, R. Folch \& J. Escarré (BC 628259); Cambrils, 23-VII-1972, R. Folch (BC 628258). Teruel: [G. Mateo in Catal. Florístico Prov. Teruel 121 (1990); Atlas Flora Aragón, http://www.ipe.csic.es/floragon/distribucion.php?cod_taxon=2405\&genero $=$ Logfia\&especie $=$ gallica\&subespecie $=\&$ variedad $=]$. Toledo: El Real de San
Vicente, 10-VI-1994, A. Segura Zubizarreta (MA 570241); entre Los Yébenes y La Dehesa Boyal, Sierra del Rebollarejo, 20-IV-2006, M.M. Martínez-Ortega ES. Andrés-Sánchez SA067 (SALA 134282); Azután, campos de cultivo, 25. V-1969, M. Ladero (MAF 82551). Valencia: Sierra de Pina, 28-VI-1886, C. Pau (MA 124302); Carcaixent, El Tossal, 28-V-1984, J.B. Peris E G. Stubing (MGC 20807, COI 68/1202). Valladolid: Bayona, 20-VI-35, B. Schafer (K). Vizcaya: Baquio, VIII-1941, E. Guinea (MA 167601); Montes de Vizcaya, A.J. Cavanilles (MA 124305). Zamora: Belver de los Montes, tesos calizos al norte del pueblo, 9-V-2005, M.M. Martínez-Ortega E S. Andrés-Sánchez MO1815 (SALA 134223); La Puebla de Sanabria, VI-1941, Losa (BCN 35658); Tábara, La Jareta, 15-VI-1996, P. Bariego Hernández (MA 650912). Zaragoza: Pinèdes de Zaragoza, 27-VI, Herbier de M. Henri Humbert (P); Cubel, 7-VII-1988, A. Martínez (JACA 426289). SWITZERLAND: Geneve, Maret, 18-IX-69, Herbarium Helveticum communicated to Sir JOSEPH D. HOOKER, 1879 (K); M. Saléve, Comm., 1821, D. Dhére (P); Vernier, près Genève, champs, 8 IX-89, P. Chenevard (GOET Old Herbarium ). TUNISIA: Oued el Hammam (Ouchteta), 25-V-1886, A. Letourneaux (P); Zembia, 26-V-53, G. Pottier-Alapetite (MPU POTTIER-ALAPETITE); Béja (gobernación), Nefta, carretera camino de la playa de Zouara, 29-III-2009, C. Aedo \& al. AQ3055 (SALA 110228). TURKEY: Cilicia, près Anamour, V-1872, A. Péronin - Pl. de Cilicie 52 (P); Constantinopla, Aucher Eloy 3125 (MPU); Smyrna, in ruderatis Smyrnae, V-1827, Fleischer - Unio itinera (GOET Old Herbarium). UNITED KINGDOM: Essex, Alresford, 25-VII-1874, E.G. Vareune 633 (K); Essex, Colchester, by Birch Grove, 26-VII-1886, E. F. Linton (K); Essex, Berechurch, 24-X-1849, O. G. Varenne 633 (P). UNITED STATES: Hawaii, Kipuka Nene (near), 6-VI-1967, O \& I. Degener 31908 (GOET); Hawaii, four miles from Chain-of-Craters Road towards Kipuka Nene, Kan Desert, Hawaii, 9-V-1966, O. E I. Degener - Plants of Hawaii (GOET); California, El Dorado County, Pine Hill. Sunny Hill Rd., 1-VIII-1997, P. Rodriguez-Rojo E D. Sánchez-Mata (MAF 158393).

2. Logfia clementei (Willk.) Holub in Bot. J. Linn. Soc. 71: 271 (1976). Filago clementei Willk. in Bot. Zeitung (Berlin) 5: 859 (1847), [basion.]. Filago mareotica subsp. clementei (Willk.) Maire in Jahand. \& Maire, Cat. Pl. Maroc 3: 747 (1934). Ind. loc.: "Spanien, Cabo de Gata, Clemente". (Lectotype, here designated, COI-WILLK COI 00035429 !; syntype: MA 124232!, MA 124233!).

Logfia dichotoma Pomel, Nouv. Mat. Fl. Atlant. 1: 44 (1874). Oglifa dichotoma (Pomel) Chrtek \& Holub in Preslia 35: 9 (1963). Filago cupaniana subsp. dichotoma (Pomel) Murb., Contr. Fl. Maroc 2: 51 (1923). Filago beterantha subsp. dichotoma (Pomel) Maire in Jahand. \& Maire, Cat. Pl. Maroc. 3: 746 (1934). Ind. loc.: "Ghar-Rhouban, Pomel". (Holotype, MPU 004847!).

Logfia willkommii Pomel in Bull. Soc. Bot. France 35: 336 (1888), nom. inval. (Art. 34.1).

Iconographies: Fig. 1b; Fig. 2 a-m.

Annual herb densely hairy, with indumentum grayish to gray-whitish. Stems 2-10 cm, branched or rarely unbranched. Leaves of the stem $6-10 \times 0.5-1.5 \mathrm{~mm}$, adpressed, linear to linear-elliptic, flat in the margin; involucrant leaves $4-7,4-5 \times$ 1.5-2 mm, similar length than the capitula, lanceolate to elliptic, with the margin straight and flat. Inflorescence in clusters arranged like to dichasium or pleochasium in the base, and like to monochasium with short branches and spiciform appearance on the top, \pm lax; clusters with 1-3 capitula, lax. Capitula 3-4 × 2-2.5 mm, sessile, with 5 marked angles. Phyllaries $1-1.5 \times 0.5-0.8 \mathrm{~mm}$, narrowly lanceolate to lanceolate, green to brownish with hyaline margin, sometimes purple at the tip, subglabrous to villose-tomentose on the abaxial face. Receptacular paleae 8-10; external 3-5, 2-3 × 0.8-1 mm, completely enclosing the external female floret, acute to subacute, 
green with hyaline margin, sometimes purple at the tip, coriaceous in fruit, villose-tomentose in the abaxial face; internal 5 $6,2-3 \times 0.8-1 \mathrm{~mm}$, surrounding together the inner florets, obtuse, green with hyaline margin, sometimes purple at the tip, scarious in fruit, glabrous to subglabrous in the abaxial face. External florets 3-5, 2-2.5 mm, female; inner florets of two types, 4-7 female similar to external florets and 4-7 hermafrodite of $2-2.5 \mathrm{~mm}$. Achenes of the external female florets 0.8-1 $\times 0.3-0.4 \mathrm{~mm}$; inner 0.6-0.8 $\times 0.3-0.4 \mathrm{~mm}$. Pappus of the internal florets with $12-25$ bristles. $2 n=$ c. 28

Habitat, phenology and distribution (Fig. 4). Ruderal, road margins, fallow lands, stony areas, terophytic pastures in forest or scrub gaps, salt marshes, dry riverbeds, limestone or chalky; 0-1700 m. III-VI. Iberian Peninsula, Canary Islands, Morocco and Algeria. SE of the Iberian Peninsula. Esp.: Al $\mathrm{GrMu}$.

Observations. Willkomm in Prodromus Florae Hispanicae (1870) proposed L. clementei and Filago ramosissima Lange as synonyms. This mistake could be caused by the fact that the sheet of F. ramosissima (COI 00035430) revised by Willkomm is a duplicate of a collection of Lange in Barranco Bermejo (Granada) and, in fact, the material lodged in this sheet corresponds to L. clementei. Moreover both taxa are macromorphologically very similar, as evidenced by the fact that Lange mixed material of both species in his own collections of F. ramosissima, as we have verified while revising the herbarium materials lodged at $\mathrm{C}$ as well as in the herbarium sheet that was sent by Lange to Willkomm.

Later, Maire (1935) and Wagenitz (1968) mentioned and cleared this mistake on the basis of morphological characters. Recent molecular studies (Galbany-Casals \& al., 2010) support this idea: they are two independent species, that even belong to two different genera.

The typification of L. clementei is also problematic. Wagenitz (1968) suggested that the possible type of this taxon could be lodged at MA. But, according to Art. 7.10 of the ICBN (McNeill, 2006), Wagenitz did not definitively typify this species because he did not checked whether the sheet collected by Clemente was effectively deposited at MA. We have found three sheets that can be considered relevant material for the typification of the name $F$. clementei, two of them lodged at MA and the other one at COI. We chose as lectotype the sheet COI 00035429 because it is included in the herbarium of the author who first described the taxon.

\section{Selected material}

ALGERIA: Ghar-Rouban, A. N. Pomel (MPU MAIRE MPU 004847). MOROCCO: Rabat-Salé-Zemmour-Zaër (Rabat), Forét de Serdjet Abbes, Zacer, Marchand, 23-IV-1927 (MA 448439); Marrakech, N. Wegrand, lehmiger Boden, 12-IV-1966, O.H. Volk 2216 (B); Plateau central, Ari Bouchenane, entre la cote 359m et Moulay Tahia, 28-V-1968, J. Mathez 4585 (MPU MATHEZ). SPAIN: Almería: Llanos de Tabernas, 9-V-1989, C. Morales \& A.B. Robles (GDAC 31062); Tabernas, base de Sierra Alhamilla, 24-IV-2008, S. Andrés-Sánchez \& al. SA185 (SALA 134259); Cabo de Gata, 1844, S. Clemente (COI 00035429, MA 124232, MA 124233). Granada: Sierra Elvira, 24-IV-1852, J. Lange [K 37378 (1) \& K 37379 (2)]; Barranco Bermejo, 19-IV1852, J. Lange (C); Barranco Bermejo, 19-IV-52, J. Lange (COI 00035430). Las Palmas: Fuerteventura, montaña alta prope Tiscamanita, 12-IV, O. Burchard 351 (K); Lanzarote, Tinajo, islote Lomitos Altos de Abajo, borde oeste, 11-II-1996, Á. Marrero E M. González-Martín (LPA 9863); Lanzarote, Tinajo, islote próximo al sur de Montaña Bermeja, 20-III-1996, Á. Marrero \& M.

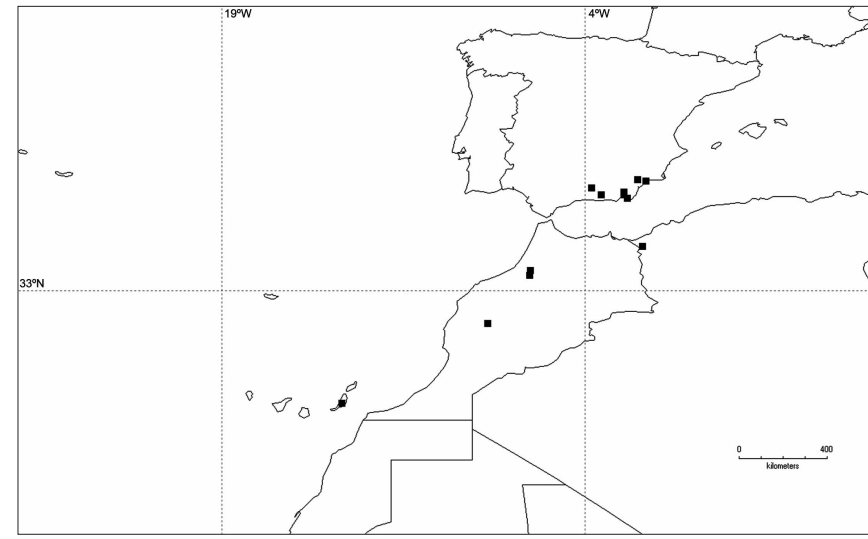

Fig. 4. Distribution map of the selected studied material corresponding to Logfia clementei.

González-Martín (LPA 18074). Murcia: Águilas, Garrobillo, cerca de Águilas, rambla del Garrobillo, 3-IV-2009, M.M. Martínez-Ortega \& S. AndrésSánchez SA259 (SALA 139202); estribaciones orientales de la Sierra del Cantar, rambla del Garrobillo, 6-V-1982, J. Molero (BCN 35801); südöstlich Lorca, bei Puerto Lumbreras, 23-IV-1967, H. Scholtz et P. Hiepko 476 (B).

3. Logfia minima (Sm.) Dumort., Fl. Belg.: 68 (1827). Gnaphalium minimum Sm., Fl. Brit. 2: 873 (1800), [basion.]. Filago minima (Sm.) Pers., Syn. Pl. 2: 422 (1807). Xerotium minimum $(\mathrm{Sm}$.$) Bluff \& Fingerh., Comp. Fl.$ German. sect. I. 2: 345 (1825). Filago montana var. minima (Sm) DC., Prodr. 6: 248 (1838). Oglifa minima (Sm.) Rchb. fil. in Rchb., Icon. Fl. Germ. Helv. 16: 27 (1853). Logfia brevifolia Cass. in F. Cuvier, Dict. Sci. Nat. ed. 2, 27: 118 (1823), nom. inval. (Art. 34.1b). Filago minima var. brevifolia (Cass.) Rouy in Rouy \& Foucaud, Fl. France 8: 176 (1903), nom. illeg. (Art. 52.1). Ind. loc.: "In arenosis et glareosis frequens". [Lectotype, here designated, LINN HS1300.59 photo!. Other original material: illustration in Petiver, Hist. Brit. II: t. 18, f. XI (1767); illustration in Morison, Pl. Hist. Univ. 3: t. 11, f. 3 (1699); illustration in Lobel, Icon. Stirp.: 481, t. 566 (1591)].

Gnaphalium montanum var. supina DC., Fl. Franç., ed. 3, 4: 136 (1805). Filago montana var. supina (DC.) DC., Prodr. 6: 248 (1838). Filago minima var. supina (DC.) Rouy in Rouy \& Foucaud, Fl. France 8: 176 (1903). (Type not found).

Iconographies: Reichenbach f. (1853: 27, lam. CMXLVI, fig. I, sub Oglifa minima); Valdés, Talavera \& Galiano (eds.) (1987: 27); Fig. 1c; Fig. 2 n-r.

Annual herb densely hairy, with indumentum grayish to gray-whitish. Stems $2-30 \mathrm{~cm}$, branched or unbranched. Leaves of the stem 1-11(20) $\times 0.5-2.5 \mathrm{~mm}, \pm$ adpressed, linear to lanceolate, flat in the margin; involucrant leaves 2-5, 2-10 $\times$ 0.5-1.5 mm, similar length than the capitula, lanceolate to elliptic, with the margin straight and flat. Inflorescence in clusters arranged like to dichasium or pleochasium, some of them like to monochasium, sometimes spiciform, usually lax; clusters with (1)3-7 capitula, lax. Capitula 2.5-4 × 1.5-3 mm, sessile or rarely short pedunculated, with 5 slightly marked angles. Phyllaries $0.5-1.5 \times 0.5-1 \mathrm{~mm}$, narrowly lanceolate to el- 
liptic, green with hyaline margin, exceptionally purple at the tip, villose-tomentose on the abaxial face. Receptacular paleae 10-12; external 4-5, 3-3.5 × 1-1.5 mm, deeply enclosing the external female floret, acute to subacute, green with hyaline margin, exceptionally purple at the tip, coriaceous in fruit although less rigid than in the other species, villose-tomentose in the abaxial face; internal 5-7, 2-3 × 0.5-1.5 mm, surrounding together the inner florets, obtuse, green with hyaline margin, exceptionally purple at the tip, scarious in fruit, glabrous to villose-tomentose in the abaxial face. External florets 4-5, 2-3 mm female; inner florets of two types, 8-15 female similar to external florets and 2-6 hermafrodite of 2-3 mm. Achenes of the external florets 0.8-1 $\times 0.2-0.4 \mathrm{~mm}$; inner 0.5-0.8 $\times$ $0.2-0.3 \mathrm{~mm}$. Pappus of the internal florets with $15-20$ bristles. $2 n=28, n=14$.

Habitat, phenology and distribution (Fig. 5): Ruderal, road margins, fallow lands and culture edges, sandy soils, forest or scrub gaps, terophytic pastures, soil indifferent; 0-2800 m. III-VIII. Europe from the Iberian Peninsula to Russia, Macaronesian Islands and $\mathrm{N}$ of Africa from Morocco to Libya. Distributed almost throughout the Iberian Peninsula. And. Esp.: $\mathrm{A} \mathrm{Ab} \mathrm{Al} \mathrm{Av} \mathrm{B} \mathrm{Ba} \mathrm{(Bi)} \mathrm{Bu} \mathrm{C} \mathrm{Ca} \mathrm{Cc} \mathrm{Co} \mathrm{CR} \mathrm{Cs} \mathrm{Cu} \mathrm{Ge} \mathrm{Gr} \mathrm{Gu} \mathrm{H}$ $\mathrm{Hu} J$ L Le Lo Lu M Ma (Mu) Na O Or P Po S Sa Sg So (SS) T Te To V Va ViZZa. Port.: AAl Ag BA BAl BB BL DL E Mi TM. N.v.: tacillas de algodón.

\section{Selected material}

ALBANIA: Bertiscus (Alpes boreales albanicae), in collibus circa oppidum Pec (Ipek), versus pag. Cruitrck, 12-VII-1933, K. Rechinger fil. E Schieffer 788 (K). ALGERIA: Orán, Aflou, champ Aitir, 16-V-88, 261 Clary, MPU MAIRE (MPU); Blida, Atlas de Blida, 23-V-1926, R. Maire (MPU MAIRE); Orán, sables, 4-VI-1845, Bedoin (MPU). ANDORRA: La Massana, track along the slope of Pic de Les Angleves, c. $2 \mathrm{~km}$. ESE of La Massana, 25-VII1998, C.C. Townsend 98/57 (K); Andorra la Vella, 2-IV-1894, M. Losa (BCN 35004). ARMENIA: Gegharkunik, Cordillera Vardenis, cerca de $4 \mathrm{~km}$ antes del paso de Selim, 23-VI-2005, E. Rico E al. AH2643 (MA 744940). AUS TRIA: Wien, Miller (MPU LITZLER); Tirol, in declioibus pr. Bagen, $V$. Grabmayr (K). Tirol occ., X-1885, Labransky (K). BELGIUM: Wesemael, 8VII-1924, E. Michel (K); Hainaut, Masmy St. Pierre, VII-1877, Herbier J. C. Fontaine 763 (P); Anvers, Grobbendonk, berge du Canal Albert, 15-VII1953, J. de Langhe (MPU LITZLER). BOSNIA-HERZEGOVINA: In Hercegovina glareosis pr. Schorzfels am Andreosbache, VIII-1871 (GOET Old Herbarium); Desbend, 8-VIII-1868, Blau 71 (K). BULGARIA: Hills S of Varna, 25-VI-25, B. Gilliart-Smith 1242 (K); 1898, Urumoff (WU-Hal-Eur); Trojan Balkan, 27-VII-1898, Urumoff (WU-Hal-Eur). CZECK REPUBLIC: Bohême, Nimes, Lorinser (ex herb. Rehsteiner) (MPU LITZLER); Raséborice (ad), VIII-1879 (WU-Hal-Eur); Moravia Orient., Bzenec (ad opp.), 12-X1925, Stan. Cermák 279 (K). DENMARK: 27, Henne, West Jutland, 16-VIII1964, Ollgaard (SALA 669); In campis prope Maunram, IX-1866, J. Lange Danish Plants (K); In gravel pit $3 \mathrm{~km}$ E of Ans, 12-VII-1972, E. Kullberg Flora Jutlandica exsiccata 729 (GOET). FRANCE: Ródano, Lyon, à Dardilly, Herbier Jordan (K). Ardèche, Peyre, 14-VI-1988, P. Litzler 88/477 (MPU LITZLER); Gard, ca. 2 km östl. Lasalle, 3-VI-1971, K. Lewejohann SF-71-254 (GOET). GERMANY: Baden, Karlsruhe, beim Flughafen, 23-VII-1963, G. Wagenitz 386 (GOET); Maingebiet, Sandgrube östl. Erlenbach; nö Meckenhard in Richtung Schippach links der StraBe, 24-VIII-1980, U. Hecker (MJG D 10235); Nieder-Sachsen, Ile de Borkum, Ostland, 21-VII-1984, J. Mennema $\&$ al. (SALA 80002). GREECE: Trigonon (Ostima), near Antartikon, 29VI-1932, A.H.G. Alston \& N.Y. Sandwith - Flora of Greece 1066 (K); Ambelakia, 25-VI-1974, Th. Raus 2384 (GOET); Macedonia occ. (distr. Peonia), inter pagos Skra et Arhangelos, 28-VII-1976, W. Greuter 13932 (GOET). HOLLAND: Noordwyk, 1844, Herb. Schultz Bip. (P); Schovenhorst, Putten, Gelderland, 28-VI-1933, P. Groenhart-Plantae Neerlandicae (K); Güeldres, Nunspect "Witsangh", 7-VIII-1960, Van Soost (don L. Rallet) (MPU LITZLER). IRELAND: Tyrone, Share of Roagh Weagh at Washing Bay SW co-

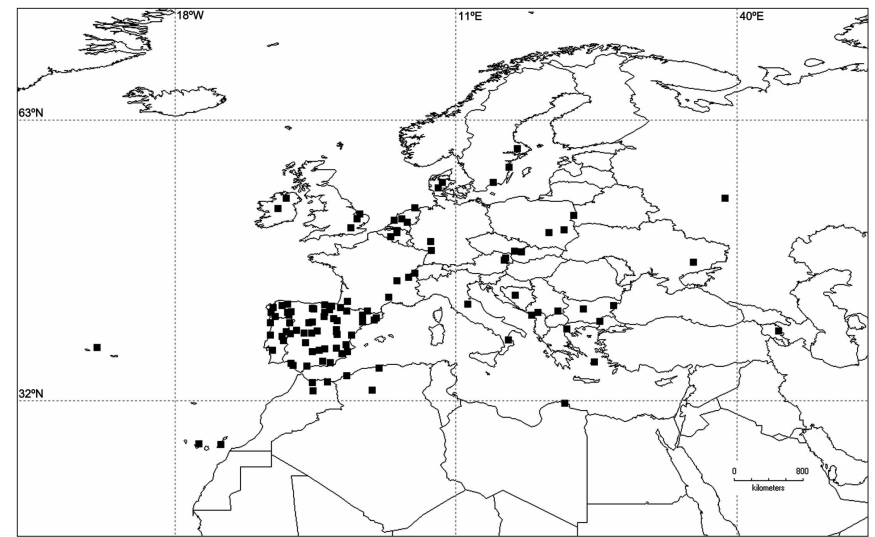

Fig. 5. Distribution map of the selected studied material corresponding to Logfia minima.

her of Lough, 1-IX-1936, Kerr 1528 (K); Loych ¿Mask?, VII-1908, Ex Herb. R. Lloyd Praeger (K). ITALY: Calabria, Sila, in ditione Longobucco, loco Fossiata dicto, 1-VIII-1918, A. Fiori 2569 (K); Prope St. Marcello, Apennino pistoriense, VI-1842, P. Savi (MPU); Appenino Bolognese, supra Porreta, Madonna del Faggio, presto Monte Acuto, 23-Juglio-1876, L. De Riva (WUHal-Eur). LIBYA: Oueu de l'oais de Dernah (Cyrenaique), VII, J. Daveau (P); Cyrenaica, $18 \mathrm{~km}$ al'ouet del'oasis de Dernah, VII-1875, Daveau - Voyage en Cyrénaïque (P). MONTENEGRO: Antivari, 1859, Herbier du Dr. Le Sourd (MPU). MOROCCO: Taza, P.N. Tazekka, subida al Jbel Tazekka, 23VI-2008, E. Rico E al. SA220 (SALA 139161); Targuist, 23-V-1927, P. Font Quer (GDA 5029); Montis du Kebdana, 14-VI-1934, Sennen \& Mauricio Plantes du Maroc (MPU MAIRE). POLAND: no Kleszczele, sw Hajnówka, 14-VIII-1976, K. Lewejohann PL-76-067 (GOET); Kielce, prope Bodzentin, 27-VII-1897, N. Puring 114 (MPU); Niemce prope Lublinum, 6-VI-1951, D. Fijalkowski (K). PORTUGAL: Algarve: Soalheira, VI-1900, Zimmermann (COI 00020669). Alto Alentejo: Mava, ¿Sadiellos?, VIII-1882, J. Henriques (COI 00020684); Marvao, junto al cruce de Baira, 28-IV-1994, E. Rico \& al. (MA 716786), Sierra de São Mamede, alrededores del pico S. Mamede, $R$. Morales \& al. RM2442 (SALA 145524). Azores: ad ruges, vias in (illegible word), VI-1838, (K). Baixo Alentejo: Distrikt Setúbal, 29 km, Luftlinie westlich von Alcácer-do-Sal bei Comporta, 26-III-1988, F. Holtz, a viagem portuguesa 05.044 (GOET). Beira Alta: estrada Sabugal-Vilar Formoso, a 3 km de Sabugal, 16-VII-1975, Fernandes, Dinis E Santos 13408 (COI 00020672); Oliveira do Hospital, Quinta da Ponte, 16-VI-1954, J. Matos, A. Matos E A. Marques 5005 (COI 00020685); Caramulo, V-1892, A. Möller (COI 00020659). Beira Baixa: Castelo Branco, $1 \mathrm{~km}$ para norte, 24-IV-1966, A. Reis Moura 660 (COI 00020706); Serra da Estrela, das mortas das servicios Florestais, 10-VIII-1954, J. Q. Abreu (MA 240706); Castelo Branco, Fundão zona alta de la sierra de la Garduña, 1-VII-2011, L. Medina \& al. LM5557 (SALA 145526). Beira Litoral: Louriçal (Arred.), Pinhal do Urso, VI-1888, M. Ferreira (COI 00020657); Aveiro, Ilhavo hacia Gafalha de Nazaré, 19-V2009, E. Rico \& al. SA296 (SALA 139159); Coimbra, Vilha Franco, V-1883, A. Möller (COI 00020687). Douro Litoral: estrada Vila do Conde - Amarante no desvio para a Touginha, a $1 \mathrm{~km}$ da Mata, 30-V-1971, C. Sergio \& M.T. Leitao 11503 (COI 00020694); Valongo, próximo ao Fojo da Valéria, 17-VI1975, Alexandre E A. Serra (PO 25227); Santo Tirso (Arred.), VII-1881, A.B. Valente (COI 00020662). Estremadura: Serra da Marofa, Cristo-Rei, 18-VII1971, A. Rozeira E al. (PO 25224). Minho: Sierra de Gerez, 27-VI-82, Gallego E al. (SEV 213194); Serra do Soajo, Senhora da Peneda, VII-1890, A. Möller (COI 00020660); Braga, Serra do Carvalho, próximo do monumento a os aviadores, 16-VI-1975, A. Dinis \& al. 13316 (COI 00020681). Tras-os-Montes: Cruce Mosqueira, 6-VII-1994, S. Castroviejo \& al. SC13223 (MA 550684); entre Amarante y Vila Real, Sierra de Marao, 28-VI-82, Gallego E al. (SEV 212969); Mogadouro, 19-V-1997, P. Montserrat \& al. (JACA 61197). RUSSIA: Rjasan, distr. Kasimov, prope pag. Naryschkino, 21-IV1973, V. Tichomirov \& al. 5893 (K). SERBIA: Vranje, hacia Golemo Selo, 21VII-2010, M.M. Martínez-Ortega \& al. SA409 (SALA 110225); Collib. Aspen ad urbical ¿Kragujevar?, 1858, Banien (P). SLOVAKIA: Trentschin, in silvis caeduis et ad silvarum margines vallis Bosacensis, Holuby 1785 (GOET); Hungaria borealis, Comit. Trentschin, in silvis caeduis et ad silvarum margines vallis Bosacensis, Holuby - Flora exsiccata Austro-Hungarica 1785 (K, P). 
SPAIN: Álava: Bernedo, Izki, Arilzulu, 24-VI-1998, M. Velayos E al. MV9202 (MA 614640); Bernedo, Urturi, 1-VII-1987, J.A. Alejandre (MA 423207); Bernedo, Izki, Arilzu, 24-VI-1998, J. L. Benito \& P. Montserrat (JACA 82998). Albacete: Tobarra, A.J. Cavanilles (MA 123788); San Pedro, 11-VI-1984, J. $M^{a}$ Herranz (MA 319560). Alicante: Cabo de Santa Pola, 5-V-1972, A. Rigual (MA 369459). Almería: Campo de Tabernas, 27-IV-1959, Hno Rufino (BCN 35806); Benizalón, cortijo de los Pajares, base de la Sierra de los Filabres, 15 IV-1988, C. Morales \& A.B. Robles (GDA 48898); Abla, 30-V-1971, J. Fernández Casas (MA 406819). Asturias: Mon, San Martín de Oscos, 8-V-1994, C. Aedo \& al. A222 (MA 539477); Cangas de Narcea, puerto del Connio, Sierra de Cazornoso, 25-VIII-2001, L. Serra \& A. Bort 6118 (MA 705649); San Pedro de la Agüera (Cerca), VII-1980, T.E. Díaz (MGC 14100). Ávila: Guisando, Nogal del Barranco, 30-V-1987, P. Vargas PV2173 (MA 655194); Navarredonda de Gredos, garganta del Jabalí, 21-V-2005, M.M. Martínez-Ortega MO1826 (SALA 134327); Navarredonda, 19-VI-1966, S. Rivas Goday (MAF 92076). Badajoz: Herrera del Duque, pastizales silicícolas de las vegas del Benazaire, 21-VI-1969, S. Rivas Goday \& M. Ladero (MAF 76827); Herrera del Duque, vegas del Benazaire, 21-VII-1969, S. Rivas Goday \& M. Ladero (MA 236314). Barcelona: La Roca del Vallés, A. de Bolós (BC 101903); Montnegre, Pla de les Garses, 1-VIII-1946, P. Montserrat (BC 618998); Montnegre, Collsacreu, marges de la carretera Arenys-Sant Celoni, 3-VII-1946, P. Montserrat (BC 618996). Burgos: Quintanar de la Sierra, 7-VII-1914, P. Font Ouer (BC 31311); Valle de Mena, Tres Concejos, Los Tueros, 24-VI-1999, P. Bariego E A. Gastón (SALA 104640); Cornubilla, Montes Obarenes, pista hacia la Casa del Austriaco, 30-V-2006, L.P. Gavilan E S. Andrés-Sánchez SA082 (SALA 134312). Cáceres: Villasbuenas de Gata, alrededores, 16-IV-1978, A. Valdés Franzi (SALA 73156); Santiago de Alcántara, Sierra de Santiago de Alcántara, 24-III-2006, E. Rico \& al. SA002 (SALA 134299); Las Hurdes, 21-V-1947, A. Caballero (MA 124211). Cádiz: Sanlúcar de Barrameda, La Algaida, 30-4-78, B. Molesworth Allen 10633 (SEV 46245); Grazalema, 15-VII-1930, L. Ceballos \& C. Vicioso (MA 124229); Grazalema, Coros, 13-V-1983, A. Aparicio E al. (SEV 213200). Cantabria: Valderredible, San Andrés de Valdelomar, 14 IX-1984, C. Aedo (MA 623562); Ormas, Hermandad de Campoo de Suso, Pico Cordel, 25-VIII-1983, C. Aedo (MA 623561); Potes, de Potes al Monasterio de Santo Toribio de Liébana, 6-VIII-1971, S. Talavera \& B. Valdés 1737/71 (SEV 11293). Castellón: Maestrat: St. Juan de Penyagolosa, El Rodesnar, 12-VII-1957, A. E O. de Bolós (BC 146869); Segorbe, Monte Malo, V1898, C. Pau (MA 124239); Canales a Andilla, 15-VI-1984, R. Figuerola E al. (MA 421804). Ciudad Real: Villamanrique, El Vizcaino, 20-V-1935, González Albo (MA 449918); Solana del Pino, Sierra Madrona, Corral de Barros, 27-VI1997, R. García Río (MA 596938); Fuencaliente, 10-V-1997, P. Montserrat (JACA 38297). Córdoba: S. ${ }^{a}$ Morena, entre Cardeña y Aldea Cerezo, 8-VI-93, M. Melendo (GDAC 41938). Cardeña a Villanueva, 10-VI-2008, C. Fernández (JAEN 80127). Cuenca: Cañete, 15-V-1978, G. López GF642 (MA 444297); La Mota del Cuervo, desvío a San Clemente, 10-V-1978, G. López GF512 (MA 449049). Gerona: supra Arbúcies, 23-V-1948, O. de Bolós (BC 104849); Alt. Empordá, Requesens, cap a la frontera, 2-VIII-1983, C. Benedí E al. (BCN 27774). Granada: Sierra de Baza, cortijo de la Almariza, 14-VI-1984, J. Torres \& al. (GDAC 25708); Jerez del Marquesado, arroyo de Alcázar, 16-VI1988, B. Valdés E al. (SEV 136384); Cáñar, Sierra Nevada, 27-VII-1930, C. Vicioso (MA 124230). Guadalajara: Vallejo Jaular, Orea, 4-VII-1986, J.M ${ }^{a} \mathrm{He}$ rranz (MA 362670); El Pedregal, arroyo de la Hoz, 13-IX-1981, D. Gómez E G. Montserrat (JACA 476681); Aldeanueva de Atienza, La Peñota, 27-VIII1966, S. Silvestre (SEV 11397). Guipúzcoa: [Aseginolaza \& al., Cat. Florístico de Álava, Vizcaya y Guipúzcoa: 756. 1984]. Huelva: Santa Bárbara, 13-V1943, C. Vicioso (MA 124228); Almonte, Matalascañas, Doñana, arenas cortafuegos cota de las Ibarras, 23-IV-1980, G. López E E. Valdés EV5453 (MA 445349); entre Higuera de la Sierra y la Yunta, 15-V-1980, J. Rivera 6614/80 (SEV 61027). Huesca: San Juan de la Peña, 14-VI-1970, J. Fernández Casas (MA 406846); Espés, Pegat, 20-VII-1988, J.V. Ferrández (JACA 450388); Benabarre, Monte Calvera, 25-VI-1987, G. Montserrat G2523.87 (JACA 609287). Jaén: Valdeazores, Despeñaperros, 28-V-1967, P. Montserrat \& F. García Novo (JACA 169267); Andújar, S. ${ }^{a}$ Quintana, 22-VI-1986, E. Cano (GDAC 28258); Andújar, Valdelagrana la Vieja, 8-VI-1985, E. Cano (MA 715113). La Coruña: río Lagüelle, Sigüeiro, 23-VII-1964, F. Bellot \& B. Casaseca (MA 200637); Doniños, a 3 km de la costa, 22-VI-1982, Gallego E al. (SEV 212965); Sigueiro, río Laguelle, 23-VII-1964, F. Bellot \& B. Casaseca (MAF 134046). La Rioja: El Sobillo, 15-VII, I. Zubia (MA 124287); Sto. Domingo de la Calzada, 20-VI, I. Zubía (MA 124219); El Rasillo de Cameros, 17 VI, I. Zubia (MA 124217). Las Palmas: Tiscamanita (prope), Fuerteventura, montaña alta, IV-12, O. Burchard - Plantae exsiccatae canarienses 351 (WU).
León: Ponferrada, Montes de Valdueza (Montes Aquilianos), 30-V-1979, E. Temprano ET222 (MA 316729); Castro del Condado, 24-VI-1969, J. Andrés (MA 569883); Soto de Valdeón, 24-VI-1978, C. García González 790 (JACA 87885). Lérida: Isona, Sierra del Cucu, Bonrepos, 4-VI-1987, P. \& G. Montserrat G1208.87 (JACA 478187); Aravell, sobre el Ulas d'Enoqueta, Alt Urgell, 10-VI-1983, J. Carreras $i$ Raurell (BCN 35017); Cadí (NW), al sud d'Ansovell, CG88, 29-VII-1977, J. Vigo \& al. (BCN 35011). Lugo: Riberas de Lea, 25-VII-1956, E. Carreira (SEV 212947); Monteseiro, Fonsagrada, VII-1957, E. Carreira (MAF 134047); Villardiaz, Fonsagrada, 20-VII-1953, E. Carreira (MA 167596). Madrid: Madrid, Campos de la Moncloa, V-1959, J. Borja (SALA 670); Madrid, 21-VI, (MAF 57550); Madrid, J.D. Rodríguez (MA 124204). Málaga: Sierra de las Nieves, 9-VII-1930, C. Vicioso (MA 124231); Tolox, P. N. Sierra de las Nieves, llano en la base del Pico Enamorados, 17VII-1997, Y. Gil \& al. (MGC 45190); Ronda, Los Alcornocales, 29-IV-1983, A. Aparicio (SEV 213201). Murcia: [Sánchez Gómez \& al., Flora de Murcia: 280, 1996]. Navarra: Garralda, 21-VII-1987, J. \& G. Montserrat (JACA 799787); Sierra de Leyre, 9-VII-1914 (MPU). Orense: Rubiá, Oulengo, Peña Tallada, 29-VI-1994, M.A. Carrasco \& al. MV7650 (SALA 115564); Lovios, sierra Santa Eufemia, 28-VI-1984, S. Castroviejo SC9267 (MA 444286); Sierra do Invernadeiro, entre Rocín y Vega de Meda, 21-VI-1973, S. Castroviejo (MA 197290). Palencia: Villabellaco, pr. Barruelo de Santullán, Los Castillos de Valle, 25-VIII-1983, C. Aedo \& al. MG858 (MA 452381); Alar del Rey, VI1936, M. Losa (BCN35652). Pontevedra: Marín, 3-VIII-1932, G. Albo (MA 124206). Salamanca: Peña de Francia, 14-VII-1927, C. Pau (MA 124225); Garcibuey, camino de la presa, 11-V-2006, L. Delgado E S. Andrés Sánchez SA079 (SALA 134279); Villarino de los Aires, 6-V-2005, L. Delgado \& M. Santos Vicente LD 937 (SALA 134221). Santa Cruz de Tenerife: Orotava, Tenerife, Los Órganos, 29-IV-1935, 502 J. Cuatrecasas E al. (MAF 62004). Segovia: Cuéllar, Casa Forestal de Fuentemacanda, 10-VI-31 (MAF 57543); Cerezo de Arriba, Pradera de Ajo, 1-VII-1983, T. Romero (MA 568424); Navares de las Cuevas, 26-VI-1983, T. Romero (SALA 39629). Soria: Matamala de Almazán, 17-VII-1958, A. Segura Zubizarreta (MA 352880); Ólvega, 18VII-1936, C. Vicioso (MA 124214); El Royo, Vega de la Razón, 3-VII-1958, P. Montserrat \& N. Y. Sandwith (JACA 47658). Tarragona: La Conga de Barberá, terme municipal Vimbodi-Poblet, Montañas de Prades, 16-VI-2009, S. Andrés-Sánchez E al. SA314 (SALA 139160); Muntanges de Prades, replá superior de la Molo de Roquerola, 28-VI-1954, F. Masclans (BC 598570); Muntanges de Prades, entre Prades i Albarca, 27-VI-1954, F. Masclans (BC598568). Teruel: Sierra de Albarracín, Guadaluviar, 6-VIII-1902, C. Pau (MA 448417); Bezas, laderas pr. Cerro Cabero, 3-VII-1995, V.J. Arán (MA 561235); Calamocha, Bea, Sierra Retuerta, 11-VII-1980, J. Molero E J.M. Martí(BC 636794). Toledo: Almorox, pinar junto a la carretera Toledo-Ávila, 20-IV-2006, M.M. Martínez-Ortega \& S. Andrés-Sánchez SA069 (SALA 134260); entre Toledo y Polán, 24-V-1968, E.F. Galiano \& al. (SEV 50995); nacimiento del río Estena, 19-VI-1986, N. Marcos \& al. MG1040 (MA 446521). Valencia: Enguera, V-1791, A.J. Cavanilles (MA 123785); La Salada, S. ${ }^{\text {de }}$ Canales, 23-VI-1911, C. Pau (MA 124238). Valladolid: Olmedo, D. Gutiérrez (MA 448418); Traspinedo-Sardón de Duero, 28-VI-1974, P. Montserrat (JACA 33074); Pozal de Gallinas, 2-VI-1957, S. Rivas Goday (MAF 01812). Vizcaya: [Aseginolaza \& al., Cat. Florístico de Álava, Vizcaya y Guipúzcoa : 756. 1984]. Zamora: Ribadelago, VI-1948, M. Losa (BCN 35804); Toro, Las Bodegas, 30-VI-1989, R. García Ríos (SALA 53040); Requejo, dans la Sierra de Cabrera, vallée du Rio Tera, a l'est de Puebla de Sanabria, 15-VII1973, J.E. De Langhe (MA 497839). Zaragoza: Illueca, 28-V-1972, A. Segura Zubizarreta (MA 352893); Sigües, Venta Carrica, 15-VI-1971, P. Montserrat E L. Villar (JACA 290071); Tabuenca, puerto de la Chavola, 13-VI-1973, L. Villar \& A. Lanaspa (JACA 178073). SWEEDEN: Blekingia, Jemshög, VII, O. Hammar (K); Upsala, 1824, Wablenberg (P); Smolandia, Loftahammar, VII-1873, N.C. Kindberg (WU-Hal-Eur). SWITZERLAND: près Bottens, Vaud, 11-VII-66, L. Favrat and W. Barbey (K); Genevam, Herb. Boissier (K); Genève, Avully, champs arides, IX-1848, Fancomet (MPU). UKRAINE: Regio sylvatica transborysthenica, in viciniis Kioviae, prope laculum Rybne dictum, 4-IX-1935, A. Lypa 190 (K). UNITED KINGDOM: Norfolk, Holkham, 11-IX-1956, C. E. Hubbard H3156 (K); Wimbledon, VI-1847, Herbier Planchon (MPU); Lakenheath, Suffolk (b. Elveden), 12-VII-1968, F.G. Schroeder (GOET).

4. Logfia heterantha (Raf.) Holub in Bot. J. Linn. Soc. 71: 271 (1976). Gnaphalium beteranthum Raf., Specchio Sci. 2: 170 (1814), [basion.]. Filago beterantha (Raf.) Guss., Fl. 
Sicul. Syn. 2: 864 (1845). Oglifa heterantha (Raf.) Pignatti in Giorn. Bot. Ital. 111: 56 (1977). Ind. loc.: "Sicilia, Palermo, Geraci Siculo, Piano Grande, zona húmeda, 3-VI2000, JGH 3225 J. Guemes \& al.”. (Neotype, here designated, SALA 106783!; isoneotype, MA 858059!, VAL 119752!, PAL 98269!).

Filago arvensis var. cupaniana DC., Prodr. 6: 249 (1838). Filago cupaniana (DC.) Parl. in Giorn. Tosc. Sci. Med. Fis. Nat. 1(2): 182 (1841). Filago beterantha subsp. cupaniana (DC.) Maire in Jahand. \& Maire, Cat. Pl. Maroc 3: 746 (1934). Ind. loc.: "in Sicilia. Gnaphalium Guss.! In litt.; Cupani ed. Bocc. t. 125". (Lectotype, here designated, G-DC G00203356 photo!).

Filago monocephala Munby in sched. (1850), nom. inval. (Art. 32.1). Type: K274248!

Logfia spicata Pomel, Nouv. Mat. Fl. Atlant. 1: 43 (1874). Ind. loc.: "Asfour sur Garrouban, Pomel". (Lectotype, here designated, MPU-Maire MPU 004848!; isotype, P 00084019!).

Iconographies. Fiori \& Paoletti (1933: 443, fig. 3537 sub Filago beterantha); Fig. $1 \mathrm{~d}$.

Annual herb densely hairy, with indumentum grayish to gray-whitish. Stems $2-30 \mathrm{~cm}$, branched or unbranched. Leaves of the stem $5-15 \times 1-2.5 \mathrm{~mm}, \pm$ adpressed, linear-elliptic to linear-spathulate, slightly undulate in the margin; involucrant leaves $2-3,3-10 \times 1-2.5 \mathrm{~mm}$, similar length to clearly longer than the capitula, lanceolate to spathulate, with the margin slightly undulate and flat to slightly involute. Inflorescense of solitary capitula, sometimes clusters, generally arranged like to monochasium with short branches and spiciform appearance, rarely like dichasium of pleochasium, \pm contracted; clusters when they are present with 2-3 capitula, lax. Capitula 3.5-5 $\times$ 2.5-4 mm, pedunculated or rarely sessile, with 5 marked angles. Phyllaries 1.5-2.8 $\times 0.5-1 \mathrm{~mm}$, lanceolate to elliptic, green to brownish with hyaline margin, never purple at the tip, villose-tomentose on the abaxial face. Receptacular paleae 10-12; external 5-7, 3-4 × 1-1.5 mm, deeply enclosing the external female floret, acute to subacute, green with hyaline margin, never purple at the tip, coriaceous in fruit, villose-tomentose in the abaxial face; internal 5-6, 1.5-3 $\times 0.8-1 \mathrm{~mm}$, surrounding together the inner florets, obtuse, green with hyaline margin, never purple at the tip, scarious in fruit, glabrous to villose-tomentose in the abaxial face. External florets 5-7, 2-3 mm, female; inner florets of two types, 3-20 female similar to external florets and 4-8 hermafrodite of $2-3 \mathrm{~mm}$. Achenes of the external female florets 0.8-1.3 $\times 0.3-0.6 \mathrm{~mm}$; inner 0.8-1.6 $\times 0.2-0.4$ $\mathrm{mm}$. Pappus of the internal florets with $15-25$ bristles.

Habitat, phenology and distribution (Fig. 6). Terophytic pastures in open areas, ruderal, forest or scrub gaps, preferably on basic substrate; 600-1900 m. V-VII. Corsica, Sardinia, Sicily and $\mathrm{N}$ of Africa from Morocco to Tunisia.

Observations. According to Stafleu \& Cowan (1983), the Rafinesque's herbarium and his types, are lodged mostly at the herbarium P-DU, although some material is also lodged at DWC, FI, G, G-DC, LE, NAP, NY, P, PH, PI, W, WIS and WS. We have sought material of Gnaphalium heteranthum Raf. in all these herbaria but we didn't succeed. For this reason we propose a neotype for this taxon. We chose a sheet

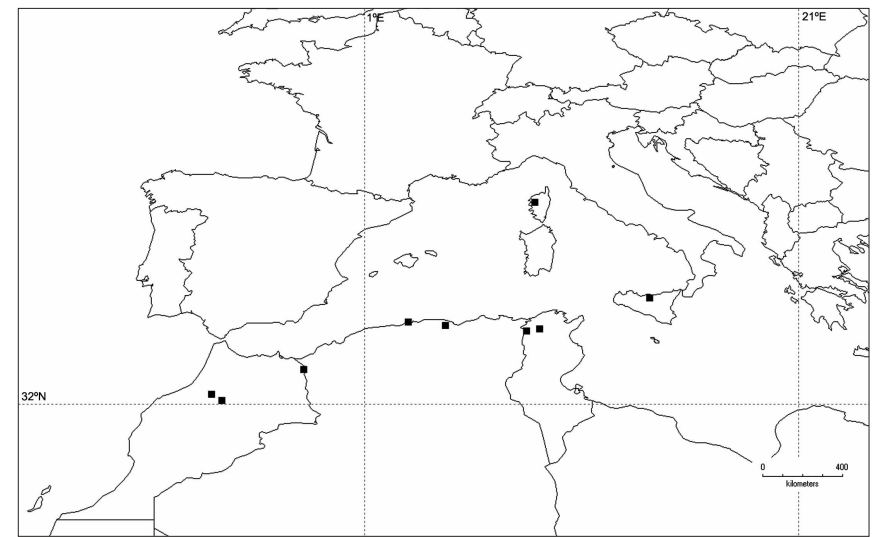

Fig. 6. Distribution map of the selected studied material corresponding to Logfia heterantha.

containing material collected in Sicily, SALA 106783, because it is the population used by Galbany-Casals \& al. (2010) for the molecular phylogenetic analyses and because this population grows near the populations cited by Rafinesque in the protologue. Finally there are duplicates of this sheet in other herbaria as MA, PAL and VAL.

\section{Selected material}

ALGERIA: Ghar-Rouban, Ras Asfour, Pomel (MPU-MAIRE MPU 004848); Alger, Le grand échatillon aus de Rouila près Alger, V, Battandier E Trabut 258 (GOET); Kabylie, Djebel Magris, VI-1898, E. Reverchon 369 (BM). FRANCE: Corse, Foret d'Aïtone, diffusa nobis, rare, 19-VI-1885, E. Reverchon (P). ITALY: Sicilia, Palermo, Geraci Siculo, Piano Grande, 3-VI2000, J. Guemes \& al. JGH3225 (SALA 106783); Sicilia, Ficuzza, 5-VI-1880, Lojacono - Plantae Siculae Rarioris 390 (WU-Hal-Eur); [Pignatti Fl. Italia 3: 35, 1982]. MOROCCO: M'rirt, 26-V-1918, R. Benoist (P); Plateau central, El-Menzeh, 10 km à l'W d'El-Khatnake, 28-V-1969, J. Mathez 4862 (MPU MATHEZ); Plateau central, Reg d'Oulmès, Arsonal, banage sur l'O Tislibad, 12-IV-1963, J. Mathez 476 (MPU MATHEZ). TUNISIA: Kef En-Nesour, Massif forestier d'El-Fedja, 20-V-1888, Cosson \& Duval (P); Djebel Ghorra, Massif forestier d'El-Fedja, 21-V-1888, E. Cosson (P).

\section{ACKNOWLEDGEMENTS}

We are grateful to J. Castillo for the careful elaboration of the drawings shown in Figure 2, which were kindly transferred from Flora iberica, and to Dr. A. Marrero who has confirmed the presence of Logfia clementei in the Canary Islands. Many thanks go also to the curators of the herbaria mentioned under Material and Methods and in the Observations section corresponding to Logfia heterantha. Regarding this species, we would like to express our deep gratitude to Dr. A. Freire-Fierro who searched for us the protologue by Rafinesque in the library of the Academy of Natural Sciences of Philadelphia (USA). This work fits within the project Flora iberica and has been supported by the Spanish Ministry of Science and Innovation (Ministerio de Ciencia e Innovación) through projects CGL2005-05471-C04-03, CGL2008-02982-C0302/CLI and CGL2009-07555 and by the Regional Government of Castilla y León (Junta de Castilla y León) through the project SA142A08. The first author was supported by a research grant (reference BES-2006-11909) financed also by the Spanish Ministry of Science and Innovation.

\section{REFERENCES}

Anderberg, A.A. 1991. Taxonomy and phylogeny of the tribe Gnaphalieae (Asteraceae). Opera Botanica 104: 5-195.

Alavi, S.A. 1983. Evax, Filago \& Logfia. In: Jafri, S.M.H. \& El-Gadi, A. (eds.), Flora of Lybia 107: 33-57. Department of Botany, Al Faateh University, Tripoli.

Andrés-Sánchez, S., Galbany-Casals, M., Rico, E. \& Martínez-Ortega, M.M. 
2011. A nomenclatural treatment for Logfia Cass. and Filago L. (Asteraceae) as newly circumscribed: Typification of several names. Taxon 60: 572-576.

Andrés-Sánchez, S., Temsch, E., Rico, E. \& Martínez-Ortega, M.M. 2013. Genome size in Filago L. (Asteraceae, Gnaphalieae) and related genera: Phylogenetic, evolutionary and ecological implications. Plant Systematics and Evolution 299: 331-345.

Battandier, J.A. 1888. Evax, Filago \& Micropus. In: Battandier, J.A. \& Trabut, L. (eds.), Flore de l'Algérie Dicotylédones: 436-443. Alger typographie Adolphe Jourdan; librairie F. Savy, Argel, Paris.

Bentham, G. 1873. Tribus IV. Inuloideae. In: Bentham, G. \& Hooker, J.D. (eds.), Genera Plantarum 2: 180-318. Lovell Reeve, London.

Bolòs, O. \& Vigo, J. 1996. Filago. In: Flora dels Països Catalans 3: 721-731. Barcino, Barcelona.

Candolle, A.P. 1837. Filago. In: Prodromus systematis naturalis regni vegetabilis 6: 247-249. Paris.

Cassini, H. 1819. Examen analytique du genre Filago de Linné. Bulletin des Sciences par la Société Philomatique de Paris 1819: 141-144.

Castroviejo, S. (coord. gen.). 1986-2012. Flora iberica 1-8, 10-15, 17-18, 21. Real Jardín Botánico, CSIC, Madrid.

Cosson, E. \& Germain E. 1843. Observations sur les genres Filago Tourn. et Logfia Cassini, et description d'une espèce nouvelle du genre Filago. Annales des Sciences Naturelles, ser. 2, 20: 283-292.

Devesa, J. 1987. Filago, Logfia, Evax \& Bombycilaena. In: Valdés, B., Talavera, S. \& Fernández-Galiano, E. (eds.), Flora Vascular de Andalucía Occidental 3: 24-31. Ketres, Barcelona.

Devesa, J. 2002. Bombycilaena, Evacidium, Evax, Filago \& Logfia. In: Valdés, B., Rejdali, M., Achhal El Kadmiri, A., Jury, S.L. \& Montserrat, J.M., Catalogue des plantes vasculaires du nord du Maroc, incluant del clés d'identification 2: 640-646. CSIC, Madrid.

Fernandes, A. \& Queirós, M. 1971. Contribution á la connaissance cytotaxonomique des Spermatophyta du Portugal 2. Compositae. Boletim da Sociedade Broteriana, serie 2, 45: 92-93.

Fiori, A. \& Paoletti, G. 1933. Iconographia Florae Italicae ossia Flora Italiana Illustrata. Tipografia Editrice Mariano Ricci, Firenze.

Galbany-Casals, M., Andrés-Sánchez, S., Garcia-Jacas, N., Susanna, A., Rico, E. \& Martínez-Ortega, M.M. 2010. How many of Cassini anagrams should there be? Molecular systematics and phylogenetic relationships in the "Filago group" (Asteraceae, Gnaphalieae), with special focus on the genus Filago. Taxon 59: 1671-1689.

Greuter W. 2008. Evacidium \& Filago. In: Greuter, W. \& von Raab-Straube, E. Med-Checklist. A critical inventory of vascular plants of the circum-mediterranean countries. 2 Dicotyledones (Compositae): 218-224. OPTIMA Secretariat, Palermo; Med-Checklist Trust of OPTIMA, Genève; Euro+Med Plantbase Secretariat, Berlin.

Holub, J. 1975. Filago, Logfia, Evax \& Bombycilaena. In: Davis, P.H. (ed.), Flora of Turkey and the East Aegean Islands 5: 101-114. Edinburgh University Press, Edinburgh.
Holub, J. 1976. Filago, Logfia, Evax \& Bombycilaena. In: Tutin, T.G., Heywood, V.H., Burges, N.A., Moore, D.M., Valentine D.H., Walters, S.M. \& Webb, D.A. (eds.), Flora europaea 4: 121-125. Cambridge University Press, Cambridge.

Linnaeus, C. 1753. Species Plantarum. Impensis Laurentii Salvii, Holmiae. Maire, R. 1935. Contributions à l'étude de la flore de l'Afrique du Nord. Fascicule 23. Bulletin de la Société d'Histoire Naturelle de l'Afrique du Nord. Algiers 26: 184-234.

McNeill, J., Barrie, F.R., Burdet, H.M., Demoulin, V., Hawksworth, D.L., Marhold, K., Nicolson, D.H., Prado, J., Silva, P.C., Skog, J.E., Wieserma, J.H. \& Turland, N.J. (eds.). 2006. International Code of Botanical Nomenclature (Vienna Code): Adopted by the Seventeenth International Botanical Congress Vienna, Austria, Juli 2005. Regnum Vegetabile 146. A.R.G. Gantner KG.

Pomel, A. 1874. Nouveaux Matériaux pour la Flore Atlantique. Librairie E. Savy, Paris \& Juillet St.-Lager, Alger.

Reichenbach f., H.G. 1853. Evax, Gifola, Oglifa \& Logfia. In: Reichenbach, L. \& Reichenbach f., H.G. (eds.), Icones Florae Germanicae en Helveticae 16: 26-28. Sumptibus Ambrosii Abel, Lipsiae.

Smoljaninova, L. 1959. Filagininae. In: Schischkin, B.K. (ed.), Flora of the USSR 25: 281-303. Science Publishers, USA.

Stafleu, F.A. \& Cowan, R.S. 1983. Taxonomic literature, vol. IV. Utrech, Netherlands.

Thiers, B. 2012. Index Herbariorum: A global directory of public herbaria and associated staff. New York Botanical Garden's Virtual Herbarium. http://sweetgum.nybg.org/ih/.

Valdés, B., Talavera, S. \& Fernández-Galiano, E. (eds.). 1987. Flora Vascular de Andalucía Occidental 3. Ketres, Barcelona.

Wagenitz, G. 1968. Weitere Beiträge zur Kenntnis der Gattung Filago (Compositae-Inuleae). Willdenowia 5: 55-66.

Wagenitz, G. 1969. Abgrenzung und Gliederung der Gattung Filago L. s.1. (Compositae-Inuleae). Willdenowia 5: 395-444.

Wagenitz, G. 1980. Bombycilaena \& Filago. In: Rechinger, K.H. (eds.), Flora des Iranischen Hochlandes und der Umrahmenden Gebirge 4: 11-27. Akademische Druck- u. Verlagsanstalt. Graz.

Watanabe, K. 2012. Index to Chromosome numbers in Asteraceae. http:// www.lib.kobe-u.ac.jp/infolib/meta_pub/G0000003asteraceae_e.

Willkomm, M. 1847. Spicilegium florae hispanicae. Botanische Zeitung. Berlin 5: 857-860.

Willkomm, M. 1870. Trib. VII. Gnaphalioideae. In: Willkomm, M. \& Lange, J., Prodromus Florae Hispanicae 2: 51-67. E. Schweizerbart'sche Verlargsbuchhandlung, Stuttgart.

Associate Editor: Juan A. Devesa Received: 30-XI-2012 Accepted: 7-II-2013 